\title{
Perspectives in Biochemistry
}

\section{Dominant Forces in Protein Folding}

\author{
Ken A. Dill \\ Department of Pharmaceutical Chemistry, University of California, San Francisco, California 94143-1204 \\ Received April 3, 1990; Revised Manuscript Received May 2, 1990
}

\begin{abstract}
$T_{\text {h }}$ he purpose of this review is to assess the nature and magnitudes of the dominant forces in protein folding. Since proteins are only marginally stable at room temperature, ${ }^{1}$ no type of molecular interaction is unimportant, and even small interactions can contribute significantly (positively or negatively) to stability (Alber, 1989a,b; Matthews, 1987a,b). However, the present review aims to identify only the largest forces that lead to the structural features of globular proteins: their extraordinary compactness, their core of nonpolar residues, and their considerable amounts of internal architecture.

This review explores contributions to the free energy of folding arising from electrostatics (classical charge repulsions and ion pairing), hydrogen-bonding and van der Waals interactions, intrinsic propensities, and hydrophobic interactions. An earlier review by Kauzmann (1959) introduced the importance of hydrophobic interactions. His insights were particularly remarkable considering that he did not have the benefit of known protein structures, model studies, high-resolution calorimetry, mutational methods, or force-field or statistical mechanical results. The present review aims to provide a reassessment of the factors important for folding in light of current knowledge. Also considered here are the opposing forces, conformational entropy and electrostatics.

The process of protein folding has been known for about 60 years. In 1902, Emil Fischer and Franz Hofmeister independently concluded that proteins were chains of covalently linked amino acids (Haschemeyer \& Haschemeyer, 1973) but deeper understanding of protein structure and conformational change was hindered because of the difficulty in finding conditions for solubilization. Chick and Martin (1911) were the first to discover the process of denaturation and to distinguish it from the process of aggregation. By 1925, the denaturation process was considered to be either hydrolysis of the peptide bond (Wu \& Wu, 1925; Anson \& Mirsky, 1925 ) or dehydration of the protein (Robertson, 1918). The view that protein denaturation was an unfolding process was
\end{abstract}

The free energy $\Delta G_{\text {unfold }}=G_{\text {denatured }}-G_{\text {native }}$ is typically $5-20$ $\mathrm{kcal} / \mathrm{mol}$ of protein, less than $(1 / 10) k T$ per residue, where $k=$ Boltzmann's constant and $T$ is temperature (Pace, 1975; Privalov, 1979). first put forward by Wu $(1929,1931)$. He proposed that native proteins involve regular repeated patterns of folding of the chain into a three-dimensional network somewhat resembling a crystal, held together by noncovalent linkages. "Denaturation is the breaking up of these labile linkages. Instead of being compact, the protein now becomes a diffuse structure. The surface is altered and the interior of the molecule is exposed" (Wu, 1929). "Denaturation is disorganization of the natural protein molecule, the change from the regular arrangement of a rigid structure to the irregular, diffuse arrangement of the flexible open chain" (Wu, 1931).

Before discussing forces, we ask: Is the native structure thermodynamically stable (the "thermodynamic hypothesis"; Anfinsen, 1973) or metastable, determined, for example, as the protein leaves the ribosome? To prove thermodynamic stability, it is sufficient to demonstrate that the native structure is only a function of state and does not depend on the process or initial conditions leading to that state. By definition, such a state would be at the global minimum of free energy relative to all other states accessible on that time scale. Experiments of Anson and Mirsky (1931) and Anson (1945) showed that hemoglobin folding is reversible as evidenced by similarities in the following properties of native and renatured protein: solubility, crystallizability, characteristic spectrum, binding to $\mathrm{O}_{2}$ and $\mathrm{CO}$, and inaccessibility to trypsin digestion. The folding of serum albumin and other proteins was shown to be similarly reversible by these coarse measures of native structure (Neurath et al., 1944; Anson, 1945; Lumry \& Eyring, 1954). It was then demonstrated that denaturation is also thermodynamically reversible for some proteins (Eisenberg \& Schwert, 1951; Brandts \& Lumry, 1963) and involved large conformational changes (Harrington \& Schellman, 1956; Schellman \& Schellman, 1958). Recent high-resolution calorimetry experiments show thermodynamic reversibility for many small single-domain globular proteins (Privalov, 1979, 1989; Santoro \& Bolen, 1988; Bolen \& Santoro, 1988; Pace, 1975 ) and also for some multidomain and coiled-coil proteins (Privalov, 1982). Reversibility was tested much more specifically by the experiments of Anfinsen et al. (1973) in which the disulfide bonds of bovine pancreatic ribonuclease were 
"scrambled" to random distributions of the 105 possible binding patterns and reacquisition of native structure and activity was observed upon renaturation (Haber \& Anfinsen, 1962; Anfinsen, 1973). The advantage of monitoring disulfide bonds is that they are uniquely trappable and identifiable. Similarly, two circularly permuted proteins refold to their original native states (Luger et al., 1989; Goldenberg \& Creighton, 1983). Therefore despite often extreme difficulties in the achievement of reversibility, the thermodynamic hypothesis has now been widely established. These experiments do not necessarily imply reversibility is completely general for other conditions, for other proteins, or even for other parts of a given protein than those monitored by the given experiment. It is clear that the folding of some proteins can be catalyzed by other assisting proteins, such as polypeptide binding or "chaperone" proteins (Rothman, 1989; Ostermann et al., 1989; Ellis, 1988; Anfinsen, 1973). Nevertheless, the existence of chaperones bears only on the rate that a protein is folded (provided the chaperone is a true catalyst) and has no bearing on the thermodynamic hypothesis, on the nature of the native state (if the native structure is otherwise reversible on the experimental time scale), or on the driving forces that cause it. The present discussion addresses only those proteins and conditions for which reversibility holds.

In this discussion of the nature of forces, it is useful to distinguish long-ranged and short-ranged forces, on the one hand, from local and nonlocal forces, on the other. The distance dependence defines the range: energies that depend on distance $r$ as $r^{-p}$ are long-ranged if $p \leq 3$ (ion-ion and iondipole interactions, for example) or short-ranged if $p>3$ (Lennard-Jones attractions and repulsions, for example). This inverse third-power dependence is the natural division because for simple pure media the integral that gives the total energy of a system diverges, according to this definition, for longranged forces and converges for short-ranged forces (Hill, 1960). For polymer chains such as proteins, segment position in the chain is also important, in addition to the range of force. "Local" interactions are those among chain segments that are "connected" neighbors $(i, i+1)$, or near neighbors, in the sequence (see Figure 1). "Nonlocal" refers to interactions among residues that are significantly apart in the sequence. Local interactions can arise from either long- or short-ranged forces, as can nonlocal interactions.

\section{(1) LONG-Ranged InTERACtions: Electrostatics}

Because acids and bases were among the earliest known denaturants of proteins, the folding forces were first assumed to be electrostatic in nature. The signature of electrostatically driven processes is a dependence on $\mathrm{pH}$ and/or ionic strength. Whereas the $\mathrm{pH}$ determines the total charge on the protein, the salt determines the extent of interaction among those charges since salts shield charges. The first quantitative model of electrostatic interactions in native proteins was proposed by Linderstrom-Lang (1924) (when he was 27 years old!). This work appeared less than 1 year after the Debye-Huckel theory on which it was based. By treating a native protein as a multivalent impenetrable spherical particle with its net charge uniformly distributed at the surface, Linderstrom-Lang predicted the number of protons bound and the net charge as functions of the hydrogen ion concentration, i.e., the $\mathrm{pH}$ titration curve. The view that protein electrostatics can be represented in terms of charges on a sphere of low dielectric constant in a higher dielectric medium has remained useful. Recent improvements have included (i) the consideration of discrete charges located at specific positions on the spherical native protein (Tanford \& Kirkwood, 1957a,b; Matthew \&
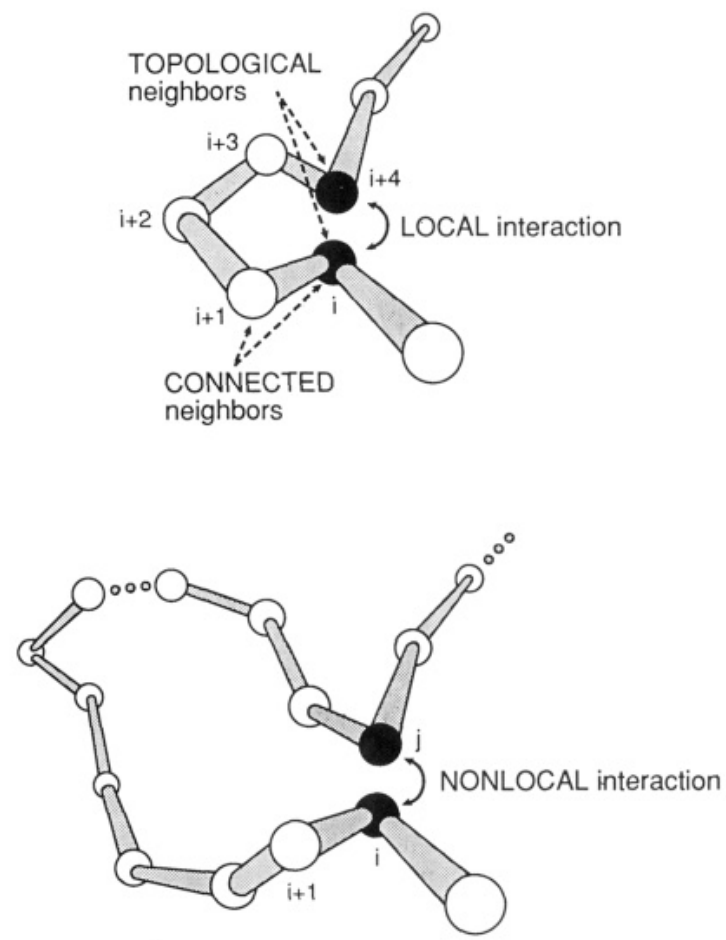

FIGURE 1: Spatially neighboring residues $(i, j)$ are defined as connected neighbors if they share a backbone bond, $j=i+1$; otherwise, they are topological neighbors. Interactions are local or nonlocal depending on the separation along the chain of the interacting residues.

Gurd, 1986; Matthew \& Richards, 1982), (ii) modeling native structural deviations from spherical shape (Gilson \& Honig, 1988a,b), and (iii) the development of electrostatic theory for the unfolded state and therefore for free energy contributions to stability (Stigter \& Dill, 1990; Stigter et al., 1990).

There are two different ways in which electrostatic interactions can affect protein stability. (1) Classical electrostatic effects are the nonspecific repulsions that arise when a protein is highly charged, for example, at extremes of $\mathrm{pH}$. The traditional view (Tanford, 1961; Kauzmann 1954; LinderstromLang, 1924) of these effects has been that the electrostatic free energy depends on the square of the net charge. Hence, no electrostatic contribution to protein stability is expected near the isoelectric point. As the net charge on the native protein is increased by increasing acidity or basicity of the solution, the increasing charge repulsion will destabilize the folded protein because the charge density on the folded molecule is greater than on the unfolded molecule. Thus, the process of unfolding leads to a state of lower electrostatic free energy. Hence, acids and bases destabilize native proteins (see Figure 2)

(2) Specific charge interactions can also affect stability. For example, ion pairing (salt bridging) occurs when oppositely charged amino acid side chains are in close spatial proximity. Whereas the classical mechanism predicts that increasing the charge could only destabilize the folded state, ion pairing could stabilize it. It has traditionally been held that classical and ion-pairing effects could be distinguished by experiments on the effects of salt concentration (below about 0.1-1.0 M) or the dielectric constant of the solution. Only at low concentrations does salt predominantly affect electrostatic shielding; at higher concentrations the electrostatic shielding is saturated, so that then the dominant effects of salt, like any other additives, are on the solvent properties of the solution (Morrison, 1952). In the traditional view, it is assumed that salts and the dielectric constant of the medium do not affect the net charge on the molecule and that they affect the native state 


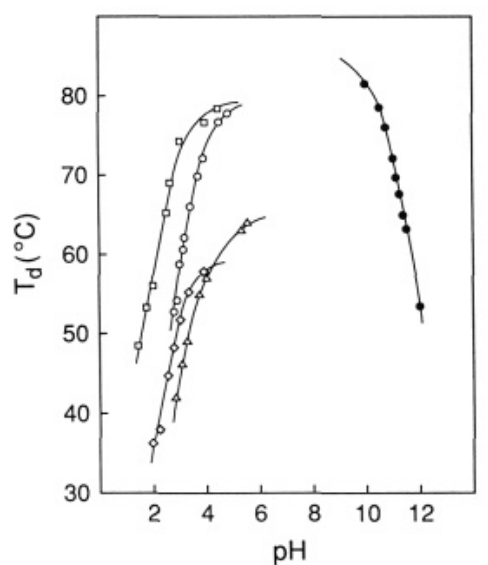

FIGURE 2: Denaturation temperature vs $\mathrm{pH}$ for $(\bullet)$ metmyoglobin, $(\Delta)$ ribonuclease $\mathrm{A},(0)$ cytochrome $\mathrm{C},(\diamond) \alpha$-chymotrypsin and $(\square)$ lysozyme. Increased charge on the protein at extremes of $\mathrm{pH}$ (low or high) favors unfolding. Reproduced from Privalov, P. L., \& Kechinashvili, N. N. (1974) J. Mol. Biol. 86, 665. Copyright (1974) Academic Press Inc. (London) Ltd.

more than the denatured state. It has often been assumed therefore that either adding salt or increasing the dielectric constant of the solution should stabilize proteins if classical effects are dominant or destabilize them if ion pairing is important. It is now clear, however, that effects of neither salt nor dielectric constant can be interpreted so simply, for the following reasons. First, salts strongly affect the unfolded state (see below). Second, ion-pair bonds are generally much shorter than the Debye lengths in salt solutions, so salt should have little effect on ion-pairing stability. [One exception is a Glu-2-...Arg- $10^{+}$salt bridge in the C-peptide helix (Shoemaker et al., 1990), which is screened by $1 \mathrm{M}$ salt, but this may be a solvent-separated ion pair (R. L. Baldwin, personal communication).] Third, although a decreased dielectric constant will lead to increased charge interactions, it will also decrease the total ionization since charging is energetically more costly in a low-dielectric medium. Moreover, the dielectric constant is also correlated with other solvent properties such as hydrophobicity and is not a simple diagnostic for charge effects alone. Therefore, discriminating between classical and ionpairing electrostatics contributions to stability has been difficult.

During the 1930 s, ion pairing was considered to be the dominant contributor to protein stability (Cohn et al., 1933; Mirsky \& Pauling, 1936; Eyring \& Stearn, 1939). Mirsky and Pauling suggested that folding was driven by the ion pairing of carboxyl and amino groups on the side chains of the charged amino acids.

If ion pairing is important for protein stability, then such stability must arise from charged pairs at protein surfaces rather than from charged pairs buried in the protein core. The first evidence that few ion pairs are buried was due to Jacobsen and Linderstrom-Lang (1949) on model compounds. An important signature of electrostatic effects in solution is a change in volume: the local volume of water decreases around a molecule of increasing charge. The electrostatic field of the charged molecule orients and orders neighboring water dipoles (electrostriction), decreasing the entropy and volume of the local water molecules. At low $\mathrm{pH}$ where only the carboxyl groups are titratable, Jacobsen and Linderstrom-Lang noted that the volume increase upon protonation of $\mathrm{COO}^{-}$to $\mathrm{COOH}$, of about $10 \mathrm{~mL} / \mathrm{mol}$ in proteins, is the same as in model carboxyl compounds in water, suggesting that ion pairs in proteins must be exposed. More recent studies of known protein structures show that indeed few ion pairs are buried

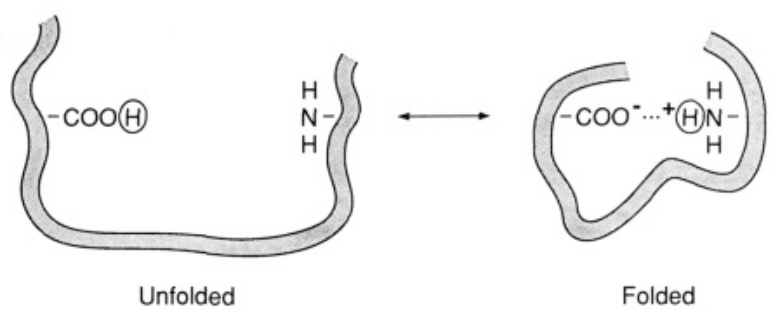

FIGURE 3: Early model in which protein folding was proposed to be driven by ion-paired hydrogen bonding among side chains (Mirsky \& Pauling, 1936; Eyring \& Stearn, 1939), shown by Jacobsen and Linderstrom-Lang (1949) to be inconsistent with partial molar volumes.

(on average, only about one ion pair per 150-residue protein is buried) (Barlow \& Thornton, 1983). This follows from the very high Born energy required to transfer a charged ion from aqueous solution to the low-dielectric interior of the protein, ranging from $19 \mathrm{kcal} / \mathrm{mol}$ for full burial to $4 \mathrm{kcal} / \mathrm{mol}$ for a half-exposed ion at the surface, $7 \mathrm{kcal} / \mathrm{mol}$ for complete burial of an ion pair (Honig et al., 1986; Honig \& Hubbell, 1984). Thus, unless other specific interactions are involved, only surface ion pairs could generally stabilize native states.

It is clear that ion pairing can contribute to protein stability. Studies of X-ray crystal structures of known proteins (Wada \& Nakamura, 1981; Barlow \& Thornton, 1983) show that ion pairing is common on the surfaces of proteins. Also, variations in sequence that affect ion pairing can change stability by about 1-3 kcal/mol of ion pairs (Fersht, 1972; Perutz \& Raidt, 1975); Asp-70-His-31 in T4 lysozyme has recently been found to stabilize by $3-5 \mathrm{kcal} / \mathrm{mol}$ (Anderson et al., 1990). Similarly, ion binding sites designed into proteins can affect stability (Pace \& Grimsley, 1988). However, it is clear that ion pairing is not the dominant force of protein folding. The first evidence emerged from the pivotal paper of Jacobsen and Linderstrom-Lang (1949). They interpreted the models of Eyring and Stearn (1939) and Mirsky and Pauling (1936) as shown in Figure 3: the hydrogen protonates the carboxyl group in the unfolded state, so both carboxyl and amino groups are uncharged, whereas in the folded state the hydrogen protonates the amino group, so that the carboxyl and amino groups form an ion pair. Jacobsen and Linderstrom-Lang presumed the charges remained solvated upon folding. Model compounds show that the protonation of $\mathrm{NH}_{2}$ to $\mathrm{NH}_{3}{ }^{+}$leads to an electrostriction of about $-4 \mathrm{~mL} / \mathrm{mol}$ and deprotonation of $\mathrm{COOH}$ to $\mathrm{COO}^{-}$leads to $-10 \mathrm{~mL} / \mathrm{mol}$, as noted above. Folding should then result in a volume change of $-14 \mathrm{~mL} / \mathrm{mol}$ per ion pair. In contrast to this model, experiments show that folding leads to an increase in volume (Jacobsen \& Linderstrom-Lang, 1949; Zipp \& Kauzmann, 1973; Brandts et al., 1970; Edelhoch \& Osborne, 1976).

Also inconsistent with ion pairing as the dominant force of folding is the observation that the stabilities of proteins show little dependence on $\mathrm{pH}$ or salt (at low salt concentrations) near the isoelectric point (Tanford, 1968; von Hippel \& Schleich, 1968; Hermans \& Scheraga, 1961; Acampora \& Hermans, 1967). [For some proteins, the $\mathrm{pH}$ of maximum stability does not coincide with the isoelectric $\mathrm{pH}$, but this can be accounted for within the classical model by the burial in the hydrocarbon core of some of the titratable groups (often histidines) (Stigter \& Dill, 1990).] As further evidence that charge generally contributes only weakly to protein stability, Hollecker and Creighton (1982) found little effect of changing the charges on several different amino groups in three different proteins. 
Third, perhaps the most persuasive evidence that ion pairing is not the dominant force of folding comes from the structural studies of Barlow and Thornton (1983). They have observed that ion pairs are not highly conserved in evolution. More importantly, the number of ion pairs in proteins is small. They observe about five ion pairs per 150 residues of protein (about one of which is buried, noted above). It is unlikely that any interaction involving only 10 residues, less than $10 \%$ of the molecule, could be the dominant folding force. Using the estimate of 1-3 kcal/mol (Fersht, 1972; Perutz \& Raidt, 1975) for the stabilization per ion pair leads to a value of 5-15 $\mathrm{kcal} / \mathrm{mol}$ stabilization. Even though ion pairing would thus contribute a free energy equal to that of the net stability of the protein, this is still $5-10$-fold smaller than the hydrophobic interaction discussed below.

A similar estimate, of about $10 \mathrm{kcal} / \mathrm{mol}$ stabilization due to ion pairing, has been made by Friend and Gurd (1979; Matthew \& Gurd, 1986). They observed decreased stability of sperm whale ferrimyoglobin with increased salt and interpreted this as evidence for ion pairing. They assumed salt predominantly affects the native state, on the basis of the difference in titration behavior of native and denatured states. However, these results do not necessarily imply the electrostatic stabilization comes from ion pairing. Salt can affect the relative free energies differently than it affects the titration behavior. A recent polyelectrolyte model of proteins (Stigter \& Dill, 1990; Stigter et al., 1990) shows instead that increasing salt, by classical effects alone, will reduce the electrostatic free energy of the unfolded state of myoglobin more than the folded state. Increased salt shields the charge repulsions in the unfolded molecule more effectively than in the folded molecule at low pH, probably because of better penetration of the salt solution into the unfolded molecule. The model is consistent with an additional experimental observation that is otherwise difficult to explain on the basis of ion pairing. For $\beta$-lactamase, similar to the myoglobin experiments of Friend and Gurd, Goto and Fink (1989) observe that salt destabilizes the native state when the molecule is highly charged at low $\mathrm{pH}$, but they also find that salt stabilizes the native structure when the molecule is charged at high $\mathrm{pH}$. It is interesting that a significant fraction of the electrostatic free energy is predicted to arise from the entropy of proton release (Stigter \& Dill, 1989, 1990), rather than simply from the charge energetics.

\section{(2) HydRogen BoNding AND VAN DER WAALS INTERACTIONS}

van der Waals attractions arise from interactions among fixed or induced dipoles. A hydrogen bond occurs when a hydrogen atom is shared between generally two electronegative atoms. Hydrogen-bond strength, which depends on the electronegativity and orientation of the bonding atoms, is in the range of $2-10 \mathrm{kcal} / \mathrm{mol}$ (Pauling, 1960). For example, the water-water hydrogen bond in the vapor phase is -6.4 $\mathrm{kcal} / \mathrm{mol}$ (Weiner et al., 1984). A hydrogen bond is primarily a linear arrangement of donor, hydrogen, and acceptor and is comprised of electrostatic, dispersion, charge-transfer, and steric repulsion interactions (Vinogradov \& Linnell, 1971). The dominant component of a hydrogen bond is electrostatic (Pauling, 1960; Cybulski \& Sheiner, 1989; Vinogradov \& Linnell, 1971). In this section we ask: do hydrogen bonds and van der Waals interactions contribute differently to folded and unfolded states of proteins, and therefore to stability? While these two types of force are microscopically quite different, there are few simple macroscopic diagnostics that can distinguish between them; hence, in this section we consider them together. The evidence cited below suggests that they may play an important role in protein folding, but the magnitudes, among all the types of force contributing to protein folding, are currently perhaps the most difficult to assess.

Mirsky and Pauling (1936) were the first to suggest that hydrogen bonding was the dominant force of protein folding. Although their focus appears to have been the electrostatic hydrogen bonds arising from ion-paired side chains (see preceding section), they also suggested that hydrogen bonding could occur between the carbonyl $\mathrm{C}=\mathrm{O}$ and amide $\mathrm{NH}$ groups of the peptide backbone. It is the peptide hydrogen bonds we consider here. Their proposal led to the X-ray crystallography studies of amino acid crystals by Pauling et al. begun in 1937 , culminating in the discovery of the $\alpha$-helix and parallel and antiparallel sheets in 1951 (Pauling et al., 1951; Pauling \& Corey, 1951a-d). These were first called "secondary structures" by Linderstrom-Lang (1952).

During the 1950s, Doty and his colleagues found a model system, poly ( $\gamma$-benzyl-L-glutamate), for studying the driving forces in the formation of polypeptide helices in nonaqueous solution (Doty \& Yang, 1956; Doty et al., 1954, 1956, 1958). Soon thereafter a theoretical framework emerged for understanding the balance of forces driving the helix-coil transition. The first theoretical model was due to Schellman (1958a). Many other elegant treatments followed, principally based on the one-dimensional Ising model (Peller, 1959; Gibbs \& DiMarzio, 1959; Zimm \& Bragg, 1959; Zimm \& Rice, 1960; Flory, 1969; Poland \& Scheraga, 1970). In these models, the intrachain hydrogen bond is considered energetically favorable relative to the hydrogen bond with the solvent. However, to form the first such bond requires overcoming configurational entropy to arrange the immediately adjacent bonds into a helical configuration. At low temperatures with simple solvents, the enthalpic contribution dominates, and the molecule forms a helix; at high temperatures, the entropy dominates and the molecule is configured as a random coil (Shoemaker et al., 1987; Marqusee et al., 1989; Lupu-Lotan et al., 1965; Platzer et al., 1972). A sharp transition between these states results from this subtle balance between the large forces. The entropy is local insofar as it involves the configurations of only immediately neighboring bonds along the chain and thus is assumed to be independent of aspects of the chain configurations more distant. Theoretical helix-coil transition models successfully predict (i) this temperature dependence and (ii) that helices become more stable and that transitions sharpen with increasing chain length (Poland \& Scheraga, 1970). The models also predict the influence of $\mathrm{pH}$ on the helix-coil transition: greater charge on the molecule destabilizes the helix since the coil has lower charge density and thus lower electrostatic free energy (Peller, 1959; Zimm \& Rice, 1960). The helix-coil transition has inverted temperature dependence in some mixed solvents (Zimm et al., 1959; Lupu-Lotan et al., 1965). Solvents that bind to the peptide bond will favor the coil; one example is formic acid, which protonates the bond (Lotan et al., 1967). Consistent with the view that hydrogen bonding is the principal driving force of the helix-coil transition, solvents that form strong hydrogen bonds compete more effectively with the peptide and destabilize the helix relative to the coil. For example, chloroform, dimethylformamide, 2-chloroethanol, trifluoroethanol, and other alcohols favor the helix, relative to formic acid, dichloroacetic acid, or trifluoroacetic acid (Doty \& Yang, 1956; Doty et al., 1954, 1956, 1958; Lupu-Lotan et al., 1965; Conio et al., 1970; Nemethy et al., 1981; Nelson \& Kallenbach, 1986). Similar theory has been developed for $\beta$-sheet formation (Mattice \& Scheraga, 1984). 
For three reasons, it is natural to assume that hydrogen bonding and van der Waals interactions will be important for the conformational changes of proteins. First, the amino acids that comprise proteins are dipolar and are capable of hydrogen bonding. Second, helices are common features of globular proteins, and the studies cited above show that the helix-coil transition is largely driven by hydrogen bonding. Similarly, intramolecular sheets are also formed by hydrogen bonding (Anufrieva et al., 1968). Third, the conformational forces for nonelectrolyte polymers in nonelectrolyte solvents are short ranged, arising from differences in monomer-monomer attractions of the chain relative to monomer-solvent attractions (Flory, 1953; deGennes, 1979). If monomer and solvent interactions are short ranged, then classical polymer theories would predict that chains should usually be relatively selfattractive, with radius changes characterized by a temperature-independent enthalpy (Flory, 1953). Such a temperature-independent enthalpy has been inferred to contribute to protein folding on the basis of model assumptions about the contribution of the hydrophobic interaction (Baldwin, 1986; Privalov, 1979; Dill et al., 1989). This residual folding force becomes more favorable as the number of polar groups increases (Privalov \& Kechinashvili, 1974). For hen lysozyme, the magnitude of this enthalpy is $0.43 \mathrm{kcal} / \mathrm{mol}$ of residues (Baldwin, 1986).

Although these short-ranged forces are therefore undoubtedly important, Kauzmann $(1954,1959)$ concluded that they are probably not the dominant forces that fold proteins in water. A fundamental criterion for a dominant driving force is that it must explain why the folded state is advantageous relative to the unfolded state. He argued that hydrogen bonding would not satisfy this criterion, because there was no basis for believing that the intrachain hydrogen bonds in the folded state would have lower free energy than those of the unfolded chain to water. In support of this view, the distribution of hydrogen-bond angles in proteins is observed to be about the same as in small-molecule compounds (Baker \& Hubbard, 1984). It follows however that folded proteins must contain many hydrogen bonds; for otherwise, the protein would denature.

Kauzmann's hypothesis led to model studies on analogues to determine the free energy of peptide hydrogen bonds in water. The several models of the peptide hydrogen bond include urea (Schellman, 1955), valerolactam (Susi, 1969), $N$-methylacetamide (NMA) (Klotz \& Franzen, 1962; Kresheck \& Klotz, 1969), and cyclic dipeptides, the diketopiperazines (Gill \& Noll, 1972). For reasons described below, however, these model studies have not yet yielded definitive estimates for the contribution of hydrogen bonds to protein stability. The dimerization binding constants and their temperature dependences have been measured for these molecules in water, in order to obtain free energies and enthalpies of dimerization. Because the concentration dependences in these experiments are linear at low concentrations, the bound species is presumed to be predominantly in the form of dimers, rather than higher multimers. At $25^{\circ} \mathrm{C}$, dimerization in water is disfavored; the equilibrium ratio of dimers to monomers is only $4.1 \times 10^{-2}$ for urea and $5.0 \times 10^{-2}$ for diketopiperazine. Thus, the free energy of dimerization is positive $\left(\Delta G_{\text {dimerization }}=+1.89\right.$ $\mathrm{kcal} / \mathrm{mol}$ for urea). However, the enthalpy of dimer formation is negative $(-2.1 \mathrm{kcal} / \mathrm{mol}$ for diketopiperazine, $-2.1 \mathrm{kcal} / \mathrm{mol}$ for urea, $-2.8 \mathrm{kcal} / \mathrm{mol}$ for valerolactam), except for $N$. methylacetamide for which it is approximately zero (Klotz \& Franzen, 1962). On the assumption that the hydrogen bond is the only attraction driving dimerization, it has been generally concluded that the hydrogen bond in water is enthalpically favored relative to the monomer-water bond. For $N$ methylacetamide, Jorgensen (1989) has shown by Monte Carlo simulation that this assumption is probably not valid: in water, the amides stack rather than form hydrogen bonds. In contrast, in chloroform, the amides form good hydrogen bonds (Jorgensen, 1989). Susi and Ard (1969) have suggested that $\epsilon$-caprolactam dimerization may also be driven by some mechanism other than hydrogen bonding. Thus in addition to hydrogen bonding, van der Waals and other interactions may also contribute significantly, but their importance for the other model compounds is not yet clear.

An additional problem prevents unequivocal determination of the free energy of hydrogen-bond formation from these model studies. In all the model compounds, there are two ways a dimer can form: singly bonded, wherein one partner in the dimer will have considerable rotational freedom relative to the other, or doubly bonded, with one partner considerably restricted in its rotation relative to the other. The experiments find only the ratio of "complexed" molecules (of singly-bonded plus doubly-bonded dimers) to unbound monomers. To obtain the free energy of hydrogen-bond formation from these data requires additional knowledge of the relative numbers of singly-bonded and doubly-bonded dimers, not currently available for these model compounds. The dilemma is illustrated by the following comparison. Suppose, on the one hand, that the only species in solution was known to be the singlybonded dimer; then the measured positive free energy implies hydrogen bonding is disfavored in water. Suppose alternatively that the only species in solution was known to be the doubly-bonded dimer. Then the binding free energy will include contributions from the two hydrogen bonds and an unfavorable entropy of rotational restriction. If this rotational restriction is sufficiently unfavorable, contributing a large enough positive free energy to the overall dimerization free energy, then the intrinsic free energy of hydrogen-bond formation will be inferred to be negative, implying that hydrogen bonding is $f a-$ vored in water. Schellman (1955) estimated this entropic restriction to be 3-6 eu and concluded that the free energy of formation of a hydrogen bond is negative but probably small. Thus the inference as to whether hydrogen-bond formation is favored or disfavored in water depends on (i) which compound is chosen as a model, (ii) the importance of interactions other than hydrogen bonding for the association processes of those model compounds in water, and (iii) estimation of the magnitude of a rotational restriction entropy, presently unknown but probably of about the same magnitude as the free energy of hydrogen bonding itself.

Moreover, this class of experiments has largely been restricted to aqueous solvents. But the peptide hydrogen bond in a globular protein is in a more hydrocarbon-like medium. We are therefore interested in the following equilibrium:

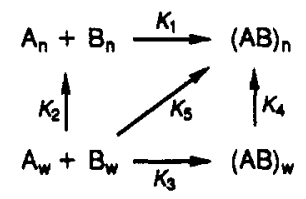

where $A$ and $B$ are the hydrogen-bond donor and acceptor, $\mathrm{w}$ is water, and $\mathrm{n}$ is the nonpolar solvent. We aim to determine $\Delta G_{5}$, the hydrogen-bond contribution to protein stability. We obtain this by using other steps of the thermodynamic cycle. (1) A wide range of hydrogen-bonding species, including NMA, formamide, alcohols, carboxylic acids, and phenols, tend to associate in nonpolar solvents $\left(K_{1}>1\right)$; for example, for the dimerization of NMA in $\mathrm{CCl}_{4}, \Delta G_{1}=-2.4 \mathrm{kcal} / \mathrm{mol}$ 
(Vinogradov \& Linnell, 1971; Klotz \& Farnham, 1968; Kresheck \& Klotz, 1969; Roseman, 1988; Sneddon et al., 1989). However, this free energy is solvent dependent. Hydrogen bonding strengthens in nonpolar solvents either if (i) the dielectric constant of the solvent is reduced, with other solvent properties held fixed (Franzen \& Stephens, 1963), as expected for Coulomb interactions, or if (ii) the electron- or proton-donating or -accepting capacity of the solvent is varied, with the dielectric constant held fixed (Allen et al., 1966; Krikorian, 1982). (2) Transferring a hydrogen bond into a nonpolar medium is generally disfavored: $\Delta G_{2}=+6.12$ $\mathrm{kcal} / \mathrm{mol}$ has been estimated for NMA from water to $\mathrm{CCl}_{4}$ (Roseman, 1988). (3) As noted above, AB dimerization is disfavored in water; for NMA, $\Delta G_{3}=+3.1 \mathrm{kcal} / \mathrm{mol}$ (Klotz \& Farnham, 1968; Roseman, 1988). (4) It follows that $\Delta G_{4}$ $=+0.62 \mathrm{kcal} / \mathrm{mol}$ for NMA in $\mathrm{CCl}_{4}$ (Roseman, 1988); $\Delta G_{4}$ is also near zero for carboxylic acids in benzene (Klotz \& Farnham, 1968) and is predicted to be $+2.2 \mathrm{kcal} / \mathrm{mol}$ for transferring the formamide dimer from water to $\mathrm{CCl}_{4}$ (Sneddon et al., 1989). (5) It follows from these estimates that hydrogen bonding opposes folding. For NMA in $\mathrm{CCl}_{4}$, $\Delta G_{5}=+3.72 \mathrm{kcal} / \mathrm{mol}$; for formamide, $\Delta G_{5}=+1.9 \mathrm{kcal} / \mathrm{mol}$. However, if the transfer of $A$ and $B$ from water into a nonpolar medium is driven by some other force, such as hydrophobicity (see below), then hydrogen-bond formation, process 1 , will be strongly favored within the folded structure. Thus whereas hydrogen bonding may not assist the collapse process, it would favor internal organization within the compact protein. Two additional uncertainties make it difficult to estimate the medium effect on the hydrogen-bond strength: (i) the nonpolar core of a protein is not a homogeneous dielectric (Honig et al., 1986; Warshel, 1984), and (ii) hydrogen-bond strength is extremely sensitive to geometric details of bond angles (Scheiner \& Hillenbrand, 1985; Scheiner et al., 1986). Since there are so many hydrogen bonds in native proteins, then even small errors in estimating their strength will lead to large errors in determining their effects on protein stability. Only $11 \%$ of all $\mathrm{C}=\mathrm{O}$ groups and $12 \%$ of all NH groups have no hydrogen bonds (Baker \& Hubbard, 1984). Of all the hydrogen bonds to $\mathrm{C}=\mathrm{O}$ groups, $43 \%$ are to water, $11 \%$ are to side chains, and $46 \%$ are to main-chain NH groups. Of all the hydrogen bonds to NH groups, $21 \%$ are to water, $11 \%$ are to side chains, and $68 \%$ are to main-chain $\mathrm{C}=\mathrm{O}$ groups. To reliably estimate the stability of a protein would therefore require model studies more accurate than about $(1 / 5) k T$ per intrachain peptide bond. For the reasons noted above, this accuracy is not yet available from the current models.

Solvent denaturation studies indicate that hydrogen bonding is not the dominant folding force (Singer, 1962; Edelhoch \& Osborne, 1976). If it were, then solvents that form strong hydrogen bonds to the peptide backbone should compete effectively and unfold the protein. Those solvents that do not affect hydrogen bonding should not affect stability. In this light, several observations are of importance. (1) It would be difficult to rationalize the observation that very small concentrations of surfactants ( $1 \%$ dodecyl sulfate, for example) unfold proteins (Tanford, 1968) since they do not destabilize helices (Lupu-Lotan et al., 1965). Moreover, the effectiveness of tetraalkylammonium salts to denature proteins depends on the number of methylene groups, indicative that it is the hydrophobic interaction, rather than hydrogen bonding, which determines stability. (2) Since the $\mathrm{C}=\mathrm{O}$ group is a strong hydrogen acceptor and the NH group is a weak donor, Singer (1962) has pointed out that the solvents useful for competing with the peptide hydrogen bond would be those which are stronger donors than $\mathrm{NH}$; the peptide bond will generally compete effectively against solvents that are weaker hydrogen acceptors than $\mathrm{C}=0$. Dioxane is only a hydrogen-bond acceptor and therefore should not denature proteins if hydrogen bonding were the dominant folding force. However, dioxane denatures proteins (Singer, 1962). (3) Alcohols are more hydrophobic than water, but they enhance helix formation (Conio et al., 1970). If hydrogen bonding were the dominant folding force, alcohols should stabilize proteins. Hence, the observation that at low concentrations they destabilize proteins is inconsistent with hydrogen bonding as the principal driving force (Hermans, 1966; von Hippel \& Schleich, 1969a,b; Parodi et al., 1973). The caveat is that alcohols have complex effects on protein stability, depending on concentration and temperature (Brandts, 1969). Finally, a particularly important comparison involves the effect on protein stability of alcohols (ethanol and propanol) vs the corresponding glycols (ethylene glycol and propylene glycol). The glycols are less hydrophobic and have more hydrogen-bond sites than the corresponding alcohols. The observation that the glycols are worse denaturants is strong evidence that hydrogen bonding is less important than the hydrophobic interaction (Tanford, 1968; Herskovitz et al., 1970; von Hippel \& Schleich, 1969a).

Mutation studies show that hydrogen-bonding groups affect stability, but by an amount which can differ considerably depending on the site and nature of the mutation (Bartlett \& Marlowe, 1987). Fersht et al. (1985) have estimated from activation free energy measurements of tyrosyl-tRNA synthetase/substrate interactions that breakage of a hydrogen bond increases the free energy by $0.5-1.5 \mathrm{kcal} / \mathrm{mol}$ for unpaired uncharged donor and acceptor or about 3.5-4.5 $\mathrm{kcal} / \mathrm{mol}$ if the donor or acceptor is charged. Site-directed mutagenesis experiments in which nonhelical proline 86 in phage T4 lysozyme is replaced by other amino acids which extend the helix and add new backbone hydrogen bonds show marginal reduction, rather than increase, in stability (Alber et al., 1988). On the other hand, side-chain hydrogen-bonding groups are found to stabilize T4 lysozyme (Alber et al., 1987a; Grutter et al., 1987). However because site-directed mutagenesis experiments measure only the total change in stability upon mutation, $\Delta \Delta G$, and not the individual molecular components of that change, then these mutations may involve more than just hydrogen bonding. For example, for one of them (Thr replacing Val-157), free energy perturbation calculations show that the added stability arises from better van der Waals interactions rather than from the difference in hydrogen-bond strength (Dang et al., 1989).

\section{(3) Local Interactions: Intrinsic Propensities}

The term "intrinsic propensity" does not describe any single type of force. Rather it is intended to convey the idea that there are certain conformational preferences of di- or tripeptides, depending on the sequence, which arise from the sum of short- and long-ranged forces that are local among connected neighboring residues. ("Local" may extend to residues three to four monomers distant and may therefore also include hydrogen bonds involved in turns or helices.) Intrinsic propensities have been studied by the measurement of helix/coil equilibria of peptides in water (Sueki et al., 1984; Marqusee et al., 1989; Marqusee \& Baldwin, 1987) and turn/coil equilibria (Dyson et al., 1985, 1988a; Wright et al., 1988). The stabilities of long polypeptide helices in aqueous solution can be attributed to intrinsic propensities. Helix stability increases with chain length (Goodman et al., 1969; Zimm \& Bragg, 1959; Poland \& Scheraga, 1970). Therefore, although the free energy contribution from each individual residue may 
be small, summed over many residues, the helix can be strongly favored relative to the coil in a long chain.

The traditional view has held that the individual residue helix/coil equilibrium constants are so nearly equal to one, however, and the initiation constants so small that short helices (less than about 15-20 residues) are not stable in aqueous solution. There have been two bases for this view. First, short helices extracted from stable globular proteins have been found to be unstable in isolation in aqueous solution (Epand \& Scheraga, 1968; Taniuchi \& Anfinsen, 1969; Dyson et al., 1988b). Second, using "guest" amino acids randomly doped into "host" copolymers of hydroxypropyl- and hydroxybutyl-L-glutamine, Scheraga and his colleagues (Sueki et al., 1984) showed that the intrinsic propensities of amino acids to form helices in water are small (with helix/coil equilibrium constants ranging from 0.59 to 1.39 at $20^{\circ} \mathrm{C}$ ). (Helical propensity can be increased considerably by reducing the temperature to near $0^{\circ} \mathrm{C}$.) However, these equilibrium constants will be universal, in principle, only if the host helix itself is completely inert in its effect on the helix/coil equilibrium of the guest residue. The following recent evidence with other hosts, however, suggests that the helix is not completely inert, i.e., that there are "context" effects. (1) The helix/coil constants differ in other sequences and can be as large as nearly 2 for alanine in alanine-based helices (Marqusee et al., 1989; Padmanabhan et al., 1990). Although the helix/coil constant for uncharged guest residue $i$ appears therefore to depend on the local sequence through residues $i-1$ and $i+1$, it does not appear to further depend on $i-2$ and $i+2$ or otherwise on the position in the chain (Merutka \& Stellwagen, 1990). (2) Additional stability results if helix formation leads to burial of nonpolar surface (Tanford, 1968; Chou et al., 1972; Richards \& Richmond, 1978). (3) Helices can be stabilized considerably by reducing the helix dipole moment through reduction of the charges at the helical ends (Shoemaker et al., 1985, 1987; Marqusee \& Baldwin, 1987). (4) Salt bridges and aromatic interactions can also affect stability (Marqusee \& Baldwin, 1987; Shoemaker et al., 1990). In addition, intrinsic propensities can vary with the solvent (Rich \& Jasensky, 1980). Recent evidence (Merutka et al., 1990) suggests that context effects may be at least as important as intrinsic propensities.

On the basis of these observations, considerable progress continues to be made in improving stabilities, so that higher helix/coil equilibrium constants are achieved, in shorter chains, and at increasing temperatures up to near room temperature (Marqusee \& Baldwin, 1987; Bradley et al., 1990). Nevertheless, intrinsic propensities, in the absence of other forces, appear to be insufficient to account for the full helical stability in globular proteins. Helices in globular proteins are short. The average length is about 12 residues, and the most probable helix length (peak of the distribution) is less than 6 residues (Kabsch \& Sander, 1983; Levitt \& Greer, 1977; Srinivasan, 1976). Yet protein helices remain $100 \%$ helical up to temperatures near the denaturation point. Other forces must therefore also be important for stabilizing helices in globular proteins. One possibility is that there may be additional "context" effects due to the environment provided by the protein interior. For example, charges are distributed in proteins so as to stabilize the helix dipole (Blagdon \& Goodman, 1975; Richardson \& Richardson, 1988). Helices with modified charges at the ends can affect protein stability (Mitchinson \& Baldwin, 1986). In principle, helices could pack in pairs, antialigned, to reduce the net dipole moment; this probably contributes little to stability, however, since the ends of helices are generally found in a high-dielectric medium at protein surfaces (Rogers, 1989; Gilson \& Honig, 1989; Presnell \& Cohen, 1989). In contrast to these effects, the protein environment may not always stabilize helices: Alber et al. (1988) found that added hydrogen-bonded helix-extending residues in T4 lysozyme had little effect or destabilized the protein.

Other evidence suggests that protein architecture does not arise principally from intrinsic propensities. First, distributions of secondary structures predicted by intrinsic propensities are inconsistent with those in known protein crystal structures. Any model of protein stability based only on local interactions would predict, as is observed in helix/coil equilibria, that longer helices should be more stable, and therefore more probable, than shorter helices. In contrast, studies of the crystal structures of globular proteins (Kabsch \& Sander, 1983; Levitt \& Greer, 1977; Srinivasan, 1976) show just the opposite: helix probability decreases monotonically with length (see Figure 11). Similarly, longer sheets are observed to be less probable than shorter sheets, for both parallel and antiparallel sheets. These discrepancies are not repaired by local factors alone. For example, it is known that helices can be terminated by "stop" residues (Kim \& Baldwin, 1984). Stop signals will not account for the data base trends, however, which are grand averages over residues, positions, and proteins, since it would then follow that most amino acids must be helix destabilizing, in contradiction to the basic premise. Moreover, even given that local interactions impart some stability to helices and turns, it is difficult to rationalize how they would give rise to sheets, which are intrinsically nonlocal. Therefore, alternative explanations for these distributions involve nonlocal factors. For example, at high densities short peptides can form stable helices in crystals (Karle et al., 1990), suggesting the importance of packing effects. It is shown in section 8 that the distributions of internal architecture can be accounted for by steric forces of nonlocal origin.

Second, attempts to predict protein structures by use of only intrinsic propensities have had limited success (Schulz \& Schirmer, 1979; Kabsch \& Sander, 1984; Argos, 1987; Thornton, 1988; Rooman \& Wodak, 1988; Qian \& Sejnowski, 1988). Success rates are about $64 \%$ when averaged over many proteins (Thornton, 1988; Rooman \& Wodak, 1988; Qian \& Sejnowski, 1988; Holley \& Karplus, 1989). Because this is considerably better than chance, it implies that intrinsic propensities are significant determinants of protein structure. However, according to Qian and Sejnowski (1988), who have used neural net methods, "no method based solely on local information is likely to produce significantly better results for non-homologous proteins." Within a given class of proteins, success rates may be higher (Kneller et al., 1990). Limitations of intrinsic propensities are also found in studies of conformations of identical pentapeptides in different proteins (Kabsch \& Sander, 1984; Argos, 1987). Kabsch and Sander found that in 6 of 25 cases one pentamer could be found in a helix whereas the identical sequence in a different protein would be in a sheet, implying that local information alone is not sufficient to fully specify the conformation in the protein. What does the $64 \%$ success rate tell us about the magnitude of the nonlocal factors missing from these prediction methods? If we assume that the conformation of any residue can be predicted with a priori success rate $p_{0}=1 / 3$ (Chou \& Fasman, 1978), as a rough estimate for classification as a helix, sheet, or other conformation, then a success rate $p$ carries an amount of information, $\langle I\rangle$ :

$$
\langle I\rangle=p \ln \left(p / p_{\circ}\right)+(1-p) \ln \left[(1-p) /\left(1-p_{0}\right)\right]
$$


Thus a success rate of $p=0.60-0.70$ implies that local factors alone account for only about $15-30 \%$ of the total information required to make a perfect prediction. This agrees with estimates (Chan \& Dill, 1990b) that the local contributions to the stability of a six-residue helix (based on a 1.05 equilibrium constant) are about $12 \%$ of the magnitude of the nonlocal steric free energy that drives helix formation, described in section 8.

\section{(4) HYDROPHOBIC EFFECT}

Following the elimination of electrostatics as a principal force of folding by Jacobsen and Linderstrom-Lang (1949), it was suggested that protein folding was driven by the aversion for water of the nonpolar residues (Linderstrom-Lang, 1952; Lumry \& Eyring, 1954; Kauzmann, 1954). The same aversion was known to drive micelle formation; it was then assumed to be due to van der Waals interactions (Debye, 1949). In two remarkably insightful papers, Kauzmann $(1954,1959)$ made the first strong case for the importance of the hydrophobic interaction in protein folding. He reasoned that the formation of one hydrophobic "bond" (which he called an "antihydrogen bond") upon folding involves the gain of a full hydrogen bond among water molecules, which should be more important by an order of magnitude than simply a change of strength of a hydrogen bond upon folding if hydrogen bonding were the dominant folding force. In support of this view, Kauzmann offered the following evidence. First, nonpolar solvents denature proteins (Singer, 1962; von Hippel \& Schleich, 1969a). According to a hydrophobic mechanism, the nonpolar solvent reduces the free energy of the unfolded state by solvating the exposed nonpolar amino acids. Second, experiments of Christensen (1952) had shown an unusual temperature dependence in which stability not only decreases at high temperatures but also decreases at low temperatures. Kauzmann observed that this cold destabilization resembles nonpolar solvation: nonpolar solutes become more soluble in water at low temperatures (Privalov \& Gill, 1988).

A considerable body of more recent evidence continues to support the view that hydrophobicity is the dominant force of folding. First, spectroscopic and high-resolution differential scanning calorimetry experiments show the resemblance of the temperature dependence of the free energy of folding and the temperature dependence of the free energy of transfer of nonpolar model compounds from water into nonpolar media (Pace, 1975; Privalov, 1979; Privalov \& Gill, 1988). Both involve large decreases in heat capacity. Second, a large number of crystal structures of proteins have become available since Kauzmann's predictions. They show that a predominant feature of globular protein structures is that the nonpolar residues are sequestered into a core where they largely avoid contact with water (Perutz et al., 1965; Chothia, 1974, 1976; Wertz \& Scheraga, 1978; Meirovitch \& Scheraga, 1980; Guy, 1985). Third, protein stability is affected by different salt species (particularly at high salt concentrations) in the same rank order as the lyotropic (Hofmeister) series (von Hippel \& Schleich, 1969a,b; Arakawa \& Timasheff, 1984); this is generally taken as empirical evidence for hydrophobic interactions (von Hippel \& Schleich, 1969; Collins \& Washabaugh, 1985; Morrison, 1952; Morrison \& Billet, 1952). From the most stabilizing (for folded ribonuclease) to the most destabilizing, the rank order of anions is found to be $\mathrm{SO}_{4}{ }^{2-}$, $\mathrm{CH}_{3} \mathrm{COO}^{-}, \mathrm{Cl}^{-}, \mathrm{Br}^{-}, \mathrm{ClO}_{4}^{-}, \mathrm{CNS}^{-}$and the rank order of cations is $\mathrm{NH}_{4}^{+}, \mathrm{K}^{+}, \mathrm{Na}^{+}, \mathrm{Li}^{+}, \mathrm{Ca}^{2+}$. The solubilities of benzene and acetyltetraglycyl ethyl ester in aqueous salt solutions increase in the same rank order. Fourth, accessible surface studies (Richards, 1977) and site-directed mutagenesis

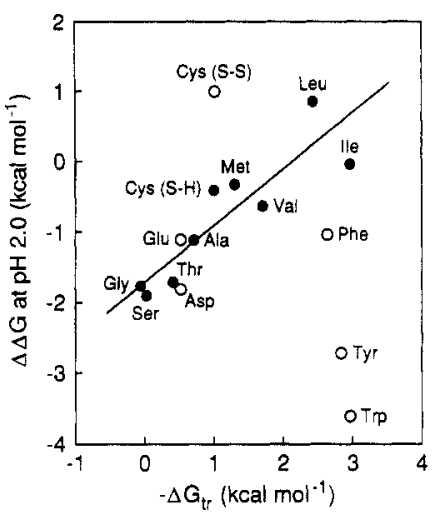

FIGURE 4: Change in free energy of unfolding, $\Delta \Delta G$, of mutant T4 lysozymes at position 3 (wild type is lle) by substitution of other residues, compared to the corresponding free energy of transfer from water to ethanol, $\Delta G_{\mathrm{tr}}$. Reprinted with permission from Matsumura, M., Becktel, W. J., \& Matthews, B. W. (1988) Nature 334, 406. Copyright (1988) Macmillan Magazines Limited.

experiments involving the replacement of a given residue by other amino acids show that the stability of the protein is proportional to the oil-water partitioning propensity of the amino acid (see Figure 4) (Yutani et al., 1984, 1987; Matsumura et al., 1988a,b; Kellis et al., 1989). Fifth, the hydrophobicities of residues in the cores of proteins appear to be more strongly conserved and correlated with structure than other types of interactions ( $\operatorname{Lim} \&$ Sauer, 1989; Bowie et al., 1990; Kelly \& Holladay, 1987; Sweet \& Eisenberg, 1983; Bashford et al., 1987). Sixth, computer simulations of incorrectly folded proteins show that the principal diagnostic of incorrect folding of proteins, apart from inappropriate burial of charge, is the interior/exterior distribution of hydrophobic residues (Novotny et al., 1984, 1988; Baumann et al., 1989).

What are "hydrophobic" interactions? There has been some disagreement about the meaning of hydrophobic (effect, force, interactions, etc.) (Hildebrand, 1968, 1979; Nemethy et al., 1968; Tanford, 1979; Ha et al., 1989). At least three different meanings of these terms have been used. (1) Hydrophobic has been used to refer to the transfer of a nonpolar solute to any aqueous solution. (2) Alternatively, it has been used more specifically to refer to transfers of nonpolar solutes into an aqueous solution only when a particular characteristic temperature dependence, described below, is observed. These two meanings describe only experimental observations and make no reference to any particular molecular interpretation. (3) "Hydrophobicity" has also been used to refer to particular molecular models, generally involving the ordering of water molecules around nonpolar solutes. In this review, hydrophobicity will be defined by (2) for reasons discussed below.

What is unusual about the temperature dependence of the hydrophobic interaction? First it is useful to describe "normal" solutions. There are two driving forces relevant to the mixing of simple solutions of $A$ with $B$, of spherical particles governed by dispersion forces. The tendency to mix is driven by an increase in the translational entropy since there are more distinguishable spatial arrangements of the A and B molecules in the mixed system than of the individual pure systems. On the other hand, mixing in simple systems is opposed by the enthalpies of interaction; ordinarily, dispersion forces leading to $A B$ attractions are smaller than those leading to the corresponding $\mathrm{AA}$ and $\mathrm{BB}$ attractions. The latter is captured in the general rule that "like dissolves like" (Hildebrand \& Scott, 1950). For simple solutions, the transfer of B from pure B into $A$ is therefore generally opposed by enthalpic interactions. When these interactions are strong, i.e., when $\mathrm{A}$ and $\mathrm{B}$ are 
relatively insoluble in each other, then the free energy of transfer is dominated by this opposing enthalpy, which is much larger than the mixing entropy, and transfer is disfavored. This is the ordinary form of incompatibility of two components $\mathrm{A}$ and $\mathrm{B}$.

Oil and water are also incompatible at $25^{\circ} \mathrm{C}$. Oil/water incompatibilities tend to be stronger than "normal" incompatibilities. However, the free energy alone (i.e., the solubility or the partition coefficient) is not the principal distinction of normal from hydrophobic processes; in both cases the transfer can be disfavored. The distinction between normal and hydrophobic processes is in the temperature dependence. What was first recognized as unusual about nonpolar transfers to water at $25^{\circ} \mathrm{C}$ (Edsall, 1935; Butler, 1937; Frank \& Evans, 1945) was that they are not principally opposed by the enthalpy; they are principally opposed by an excess (i.e., "unitary") (Gurney, 1953; Tanford, 1970) entropy (Gill \& Wadsö, 1976; Tanford, 1980; Privalov \& Gill, 1988). The excess entropy is that which remains after the mixing entropy (the $R T \ln x$ term in the chemical potential) is subtracted from the total measured entropy. The enthalpy of mixing oil and water is generally small, sometimes even negative (favorable), at $25^{\circ} \mathrm{C}$ (Privalov \& Gill, 1988). Because these conclusions derive from experiments with solutes at high dilution, they imply that the excess entropy must arise from the water solvation around the solute rather than from some possible solute-solute interaction. A molecular interpretation of these data, which is supported by computer simulations (Geiger et al., 1979; Pangali et al., 1982; Ravishanker et al., 1982), is that at $25^{\circ} \mathrm{C}$ nonpolar solutes are surrounded by ordered waters. Waters surrounding the nonpolar solute prefer to hydrogen bond with other waters rather than to "waste" hydrogen bonds by pointing them toward the nonpolar species (see top of Figure 6) (Stillinger, 1980; Geiger et al., 1979).

However, the aversion of nonpolar solutes for water becomes more ordinary, and less entropy driven, at higher temperatures. This is because there is a second fundamental difference between simple incompatibility on the one hand and oil/water incompatibility on the other. For simple solutions, the heat capacity change upon transfer is small. For nonpolar solutes, the heat capacity change upon transfer from the pure liquid to water is large and positive (Frank \& Evans, 1945; Christian \& Tucker, 1982; Privalov \& Gill, 1988; Edsall \& McKenzie, 1983). This means that for simple solutions the transfer of $A$ into $B$ is characterized by an enthalpy and entropy which are temperature independent and a free energy which is constant or linear with temperature. However, the situation is quite different for the transfer of nonpolar solutes into water. A large heat capacity implies the enthalpy and entropy are strong functions of temperature, and the free energy vs temperature is a curved function, increasing at low temperatures and decreasing at higher temperatures. Hence, there will be a temperature at which the solubility of nonpolar species in water is a minimum (Crovetto et al., 1982; Becktel \& Schellman, 1987; Edsall \& McKenzie, 1983; Privalov \& Gill, 1988) (see Figure 5). A striking consequence follows, one which is at variance with the view that water ordering is the principal feature of the aversion of nonpolar residues for water. The free energy of transferring nonpolar solutes into water is extrapolated to be most positive in the temperature range $130-160^{\circ} \mathrm{C}$ (Privalov \& Gill, 1988). Therefore, the aversion of nonpolar species for water, whatever its molecular nature, is greatest at these high temperatures. Because this maximum aversion, by definition, arises where the free energy of transfer is a maximum, and thus where the entropy (the temperature

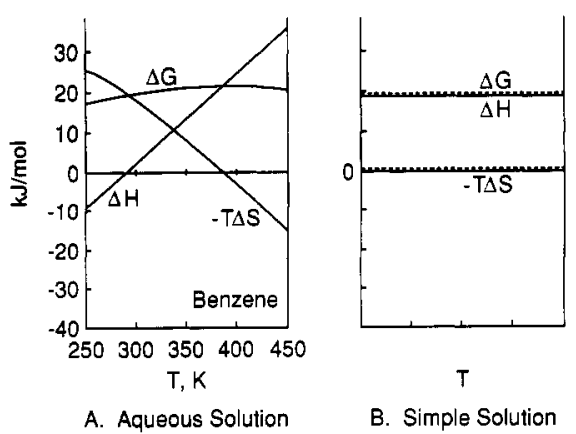

FIGURE 5: Comparison of the enthalpy $(\Delta H)$, entropy $(\Delta S)$, and free energy $(\Delta G)$ of solute transfer from the pure liquid into a hypothetical regular solution and into an aqueous solution. The data in (A) are for benzene, from Privalov and Gill (1988); the entropy at high temperature is extrapolated on the basis of assumed constant heat capacity. The figure on the right represents an idealization according to regular solution theory. Reprinted with permission from Privalov, P. L., \& Gill, S. J. (1988) Adv. Protein Chem. 39, 191. Copyright (1988) Academic Press.

derivative of the free energy) equals zero, then the maximum aversion of nonpolar solutes for water must be driven by enthalpy (Privalov \& Gill, 1988; Baldwin, 1986). In other words, at the temperature for which hydrophobicity is strongest, the entropy of transfer is zero! At those temperatures, the aversion of nonpolar solutes for water is enthalpic, as in simple classical solutions. It is therefore inappropriate to refer to nonpolar solvation processes in water, with large heat capacity changes, as "entropy driven" or "enthalpy driven", since either description is only accurate within a given temperature range. At $25^{\circ} \mathrm{C}$, the hydrophobic effect is entropic; at $140^{\circ} \mathrm{C}$, it is extrapolated to be enthalpic (Privalov \& Gill, 1988).

For processes of constant $\Delta C_{p}$, the enthalpy and entropy of transfer are

$$
\begin{gathered}
\Delta H(T)=\Delta H\left(T_{1}\right)+\int_{T_{1}}^{T} \Delta C_{p} \mathrm{~d} T= \\
\Delta H\left(T_{1}\right)+\Delta C_{p}\left(T-T_{1}\right) \\
\Delta S(T)=\Delta S\left(T_{2}\right)+\int_{T_{2}}^{T} \Delta C_{p} / T \mathrm{~d} T= \\
\Delta S\left(T_{2}\right)+\Delta C_{p} \ln \left(T / T_{2}\right)
\end{gathered}
$$

and therefore the free energy of transfer is

$$
\begin{aligned}
& \Delta G(T)=\Delta H-T \Delta S= \\
& \Delta H\left(T_{1}\right)-T \Delta S\left(T_{2}\right)+\Delta C_{p}\left[\left(T-T_{1}\right)-T \ln \left(T / T_{2}\right)\right]
\end{aligned}
$$

These quantities are defined in terms of two arbitrary reference temperatures: $T_{1}$, for which the enthalpy is known, and $T_{2}$, for which the entropy is known. There are three alternative ways to express this free energy in terms of two particularly convenient reference temperatures, $T_{\mathrm{h}}$, the temperature at which the enthalpy is zero, and $T_{\mathrm{s}}$, the temperature for which the entropy is zero:

$$
\begin{gathered}
T_{1}=T_{\mathrm{h}} \quad T_{2}=T_{\mathrm{s}} \\
\Delta G(T)=\Delta C_{p}\left[\left(T-T_{\mathrm{h}}\right)-T \ln \left(T / T_{\mathrm{s}}\right)\right] \\
T_{1}=T_{2}=T_{\mathrm{s}} \\
\Delta G(T)=\Delta H\left(T_{\mathrm{s}}\right)+\Delta C_{p}\left[\left(T-T_{\mathrm{s}}\right)-T \ln \left(T / T_{\mathrm{s}}\right)\right] \\
T_{1}=T_{2}=T_{\mathrm{h}} \\
\frac{\Delta G(T)}{R T}=-\frac{\Delta S\left(T_{\mathrm{h}}\right)}{R}+\frac{\Delta C_{p}}{R}\left[\left(1-T_{\mathrm{h}} / T\right)-\ln \left(T / T_{\mathrm{h}}\right)\right]
\end{gathered}
$$




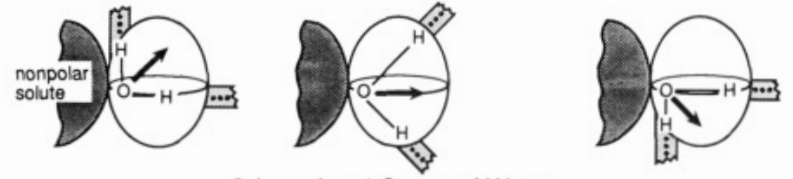

Orientational States of Water

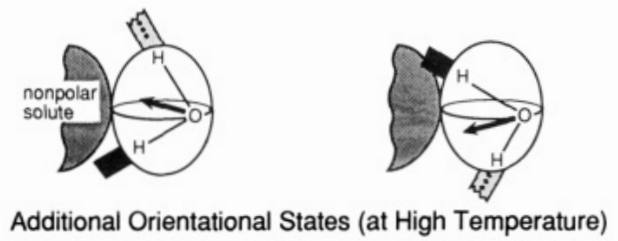

FIGURE 6: "Iceberg" model for the large heat capacity of transfer of nonpolar solutes into water (Frank \& Evans, 1945; Gill et al., 1985). At low temperatures (near room temperature for benzene, for example), the water molecules surrounding the nonpolar solute adopt only a few orientations (low entropy), to avoid "wasting" hydrogen bonds; thus, all water configurations are fully hydrogen bonded (low energy). At higher temperatures, more conformations are accessible (higher entropy), but some of them have weaker or unformed hydrogen bonds and/or van der Waals interactions (higher energy). This contributes to the heat capacity because the system energy increases with temperature.

These three expressions have identical content. Which form is used depends on which set of parameters is most convenient: $\left(\Delta C_{p}, T_{\mathrm{s}}, T_{\mathrm{h}}\right),\left(\Delta C_{p}, T_{\mathrm{s}}, \Delta H\left(T_{\mathrm{s}}\right)\right)$, or $\left(\Delta C_{\mathrm{p}}, T_{\mathrm{h}}, \Delta S\left(\mathrm{~T}_{\mathrm{h}}\right)\right)$. Ordinary thermodynamic convention is to choose a single reference temperature, rather than two, but the mixed expression is included here because it has been used for protein folding (see below). Equations 5-7 predict the type of temperature dependence shown in Figure $5 \mathrm{~A}$ when $\Delta C_{p}$ is large or in Figure 5B when $\Delta C_{p}=0$.

What is the molecular basis for the large heat capacity of transfer of nonpolar solutes into water? Two observations have contributed significantly to a molecular picture. First, the entropy and heat capacity of transfer are linearly proportional to the surface area of the nonpolar solute (Miller \& Hildebrand, 1968; Gill et al., 1985; Jorgensen et al., 1985; Jolicoeur et al., 1986). Second, the large heat capacity of transfer for the simplest solutes (Gill et al., 1985) decreases slightly with increasing temperature. This leads to the view (Frank \& Evans, 1945; Nemethy \& Scheraga, 1962; Gill et al., 1985; Muller, 1990) that the organization of water molecules in the first shell surrounding the solute is like an "iceberg", a clathrate, or a "flickering cluster"; see Figure 6. At room temperature, the water molecules surrounding the nonpolar solute principally populate a low-energy, low-entropy state: the waters are ordered so as to form good water-water hydrogen bonds. With increasing temperature, the waters surronding the solute increasingly populate a higher energy, higher entropy state: they are less ordered and have weakened attractions. Hence, increased temperature causes "melting" of the surrounding water structure, insofar as the entropy and energy are increased. [This melting process is probably better represented as bent hydrogen bonds than broken bonds, since the bending energy is much smaller than the breaking energy (Lennard-Jones \& Pople, 1951).] The reason this results in a large heat capacity is that the two different energetic states of water provide an energy storage mechanism. The higher energy state becomes more populated with temperature. The reason this heat capacity is so large per solute molecule is because each solute molecule is surrounded by a large number (more than 10) of first-shell water molecules, each of which can participate in this energy storage mechanism. Not yet known is the detailed breakdown of the nature of these energies, although they undoubtedly include some combination of solute-water and water-water hydrogen bonding and van der Waals and dipolar electrostatic interactions.

What is the molecular interpretation of $T_{\mathrm{s}}$ and $T_{\mathrm{h}}$ ? Equations 2-7 each have temperature-dependent and temperature-independent terms. The slope of the temperature dependence in eqs 5-7 is given by $\Delta C_{p} . T_{\mathrm{s}}$ and $T_{\mathrm{h}}$ can be interpreted as representing a reference enthalpy or entropy at some given temperature. $T_{\mathrm{s}}$ and $T_{\mathrm{h}}$ are diagnostic for liquid-state nonpolar transfer processes (Baldwin, 1986). Sturtevant (1977) observed that several different biomolecular processes at $25^{\circ} \mathrm{C}$ have nearly identical values of the ratio $\Delta \mathrm{S} / \Delta C_{p}$. Baldwin (1986) showed that the constancy of this ratio, taken together with $T_{2}=T_{\mathrm{s}}$ and $T=298 \mathrm{~K}$, substituted into eq 3 implies that these various processes can all be characterized by a single temperature, $T_{s}=114^{\circ} \mathrm{C}$. Using the data of Gill and Wadsö (1976) for $\Delta S$ and $\Delta C_{p}$ for the transfer of liquid nonpolar compounds to water at different temperatures, $T$, substituted into eq 3 , Baldwin again found a single characteristic temperature, $T_{\mathrm{s}}=112.8^{\circ} \mathrm{C}$, implying that Sturtevant's biomolecular processes resemble nonpolar solvation. Baldwin (1986) preferred the use of eq 5. For eqs $5-7, T_{\mathrm{s}}$ and $T_{\mathrm{h}}$ are convenient reference quantities because $\Delta H$, $\Delta S$, and $\Delta C_{p}$ depend linearly on solute surface area, so ratios such as $\Delta S / C_{p}$, and hence $T_{s}$, should be independent of solute size.

On the other hand, Murphy, Gill, and Privalov (Murphy et al., 1990; Privalov \& Gill, 1988) have preferred the convention given by eq 6 . They assumed that at temperature $T_{\mathrm{s}}$ nonpolar solvation is identical with classical solvation and that $\Delta H\left(T_{\mathrm{s}}\right)$ is due only to van der Waals forces. They refer to the factors other than $\Delta H\left(T_{\mathrm{s}}\right)$ in eq 6 as the "hydration effect". They note that $\Delta H\left(T_{\mathrm{s}}\right)$ is a positive enthalpy disfavoring transfer and that the hydration term is always negative (or zero at $T=T_{\mathrm{s}}$ ). It follows that the hydration effect favors nonpolar transfers into water. However, it is not clear that this separation into these molecular factors is warranted. At $T=T_{s}$, nonpolar solvation is not identical with simple solvation. Even though the entropy of transfer may extrapolate to zero at $T=T_{\mathrm{s}}$, the heat capacity remains large (Muller, 1990). The heat capacity is probably a more fundamental characteristic of nonpolar solvation than the entropy, because the entropy depends on the choice of concentration units, as do the free energy and partition coefficient, whereas that heat capacity and enthalpy do not. In addition, these thermodynamic models predict that for $T>T_{\mathrm{s}}$ the entropy of transfer becomes positive, leading to the questionable prediction that the entropy would then favor solvation at high temperatures (see Figure 5A for benzene above $400 \mathrm{~K}$ ). Also, since water hydrogen bonding persists to beyond $500 \mathrm{~K}$ (Crovetto et al., 1982; Franks, 1983), $\Delta H\left(T_{\mathrm{s}}\right)$ probably includes water-water hydrogen bonding in addition to van der Waals interactions. Thus, it is not clear that this thermodynamic separation of terms corresponds to a simple molecular picture. Equation 7 provides yet a different view. Whereas $\Delta G$ for transferring benzene to water is most positive at $T=T_{\mathrm{s}}\left(\right.$ near $100^{\circ} \mathrm{C}$ ), $\Delta G / R T$ is most positive at $T=T_{\mathrm{h}}$ (near room temperature). $\Delta G / R T$ corresponds directly to a solubility, partition coefficient, or Boltzmann population. Benzene is least soluble in water around room temperature. In eq $7,-\Delta S\left(T_{\mathrm{h}}\right) / R$, representing water ordering, is the most positive contribution to $\Delta G / R T$. The remaining terms, whatever their molecular interpretation, favor solvation. Thus at present, $T_{\mathrm{h}}$ and $T_{\mathrm{s}}$ serve to identify nonpolar transfer processes, but their breakdown into molecular components awaits further theory and experiments. 
The thermal unfolding of proteins shows important similarities and important differences in comparison with the solvation processes of small nonpolar solutes. The similarity is that protein unfolding involves a large increase in heat capacity, ${ }^{2}$ characteristic of nonpolar exposure (Pace, 1975; Privalov, 1979; Baldwin, 1986; Becktel \& Schellman, 1987; Schellman, 1987; Privalov \& Gill, 1988; Ooi \& Oobatake, 1988). Therefore, the enthalpy and entropy of folding strongly decrease with temperature, and the free energy is curved: maximum protein stabilities are in the range of $0-30^{\circ} \mathrm{C}$ (see Figure 8). The heat capacity of protein unfolding is itself approximately independent of temperature, although it appears to decrease somewhat at higher temperatures (Becktel \& Schellman, 1987; Gill \& Privalov, 1988).

The thermal unfolding of proteins differs from nonpolar solvation in the particular values of enthalpy and entropy at any given temperature. For example, as noted above, at 25 ${ }^{\circ} \mathrm{C}$, the transfer of small nonpolar compounds into water has approximately zero enthalpy change and a large negative excess entropy change. In contrast, at $25^{\circ} \mathrm{C}$, protein unfolding has a positive enthalpy change (except for myoglobin for which the enthalpy is about zero) and a small or positive excess entropy (Baldwin, 1986; Privalov \& Gill, 1988). There is an additional positive entropy and enthalpy of unfolding, in excess of that predicted from nonpolar solvation experiments.

What is the origin of the residual enthalpy and entropy of folding? As noted in section 2, the residual enthalpy of unfolding becomes more positive with increased content of polar residues (Privalov, 1979; Privalov \& Gill, 1988; Dill et al., 1989). If it is assumed to be approximately temperature independent (Baldwin, 1986), then this result is expected from classical polymer solution behavior wherein chain monomers of type $\mathrm{A}$ have normal dispersion-force-driven incompatibilities with solvent B (Flory, 1953). This enthalpy may arise from van der Waals or hydrogen-bonding interactions among the backbone or polar residues. However, thermodynamic models of protein unfolding have generally been based on the assumption that the large heat capacity change is fully attributable to nonpolar solvation. This is open to question. Hydrogen bonding to water weakens with temperature, according to valerolactam dimerization and thymine dissolution experiments (Alvarez \& Biltonen, 1973); this would also contribute to a change in heat capacity. Also, increased temperature causes the unfolded states of proteins to expand, reducing nonpolar solvation and contributing additional temperature dependence to the enthalpy and entropy of folding (Dill et al., 1989).

The large residual entropy represents an increased disordering upon unfolding, relative to that expected for nonpolar solvation. In sections 5 and 6 , it is suggested that at least a large component of this is due to a difference in the freedom of configurations of the chain backbone, more severely restricted by steric constraints in the folded than in the unfolded states. However, there may also be an extra residual entropy due to additional configurational restrictions of the side chains in the folded state. The side chains may be partially frozen if the folded protein resembles a solid-like state (Shakhnovich \& Finkelstein, 1989a,b). The residual entropy of protein denaturation is $\Delta S_{\mathrm{r}}\left(112^{\circ} \mathrm{C}\right)=18 \mathrm{~J} /(\mathrm{K} \cdot \mathrm{mol})$ (Baldwin, 1986; Privalov, 1979; Murphy et al., 1990), similar to that of the dissociation of solid diketopiperazines, $\Delta S_{\mathrm{r}}\left(112^{\circ} \mathrm{C}\right)=16$ $\mathrm{J} /(\mathrm{K} \cdot \mathrm{mol})$, and considerably different than that of liquid

\footnotetext{
${ }^{2}$ One interesting counterexample, however, is a highly stable phosphoglycerate kinase from a thermostable bacterium (Nojima et al., 1977) in which both the enthalpy and heat capacity of folding are small.
}

hydrocarbon dissolution for which $\Delta S_{\mathrm{r}}\left(112^{\circ} \mathrm{C}\right)=-0.5 \mathrm{~J} /$ (K·mol) (Murphy et al., 1990). The heat capacity change increment upon dissolution of the solid diketopiperazines is the same as for the liquid-state transfer process (Murphy \& Gill, 1989a,b). From those thermodynamic experiments, however, it is not possible to determine how much of the entropy difference originates with the chain expansion and solvation and how much originates from side-chain unfreezing. Bendzko et al. (1986) have suggested that if side-chain freezing is important, then protein denaturation should lead to an increase in partial molar volume of the protein; instead, they and others (see section 5) find a decreased volume upon unfolding. Another test of side-chain restrictions in the folded core is to compare crystal structure distributions of side-chain rotamers (Ponder \& Richards, 1987) with computer simulations of the mean position and fluctuations of side chains that are attached to spatially unconstrained backbones. Such comparisons (Janin et al., 1978; Piela et al., 1987) show that equilibrium side-chain positions are predicted relatively well by the unconstrained simulations, but they are constrained somewhat differently in different secondary structures (Piela et al., 1987; McGregor et al., 1987).

Another class of experiment bears on the issue of the solid-like vs liquid-like nature of the protein core. Whereas the core is solid-like in its density and compressibility, it may behave differently insofar as the transfer process is concerned. The experiments involve multiple amino acid substitutions at a given site and the measurement of the change in protein stability, $\Delta \Delta G$, due to each of the replacements (Yutani et al., 1984, 1987; Matsumura et al., 1988a,b; Kellis et al., 1989; Sandberg \& Terwilliger, 1989). The slope of $\Delta \Delta G$ vs the free energy of transfer $\Delta G_{\mathrm{tr}}$ for the corresponding amino acid from water to oil (see Figure 4), provides information about similarities and differences of the protein folding process relative to the simpler process of amino acid transfer from water into liquid oil. This slope depends on a combination of factors: (i) the "deformability" of the native-state cavity, the energetic and entropic constraints affecting the freedom of the cavity wall residues to move to accommodate the mutated amino acid; (ii) the interactions, $w$, of the residue with the cavity, including both its entropic restrictions (side-chain freezing) and the residue/cavity energetics; (iii) the degree to which the denatured-state environment of the specified residue resembles pure solvent; and (iv) the exposure of the residue in the native state. A slope of 1 is consistent with a process in which the amino acid is exposed in the unfolded state and is transferred into a native cavity that resembles the reference liquid oil. Simple solute transfer to a liquid involves (a) opening the cavity (unfavorable by approximately free energy $w / 2$ ) and then (b) transferring the solute (favorable by a free energy, $w$ ). On the other hand, if a solute is transferred instead into an already opened cavity ("preformed"), then only (b) is involved (Kellis et al., 1989). Thus, the free energy of transfer into a simple liquid is only half that of transfer into a preformed cavity. Therefore, a slope of 2 is expected from these experiments if the cavity wall residues are so constrained that they do not move to accommodate the mutant residue. Protein cavities may differ. Some may have much "deformability", as in a liquid, and lead to slopes near 1. Others may have little deformability, if neighboring residues are constrained, and lead to slopes near 2. Cavity deformabilities may also depend on residue size; cavity wall residues may move to accommodate large occupants but not small ones, for example. Overall, slopes ranging from 1 to 2 are expected from factor (i). Slopes smaller than 1 imply that the residue may be exposed in the 
native state or may be significantly buried in the denatured state. Slopes considerably greater than 2 must arise at least partly from (ii). A negative slope could arise in principle if the residue is more exposed at the surface of the native structure than it is in the denatured state. A slope of approximately 1 is observed for residue 3 in T4 lysozyme (Matsumura, 1988a). A slope of approximately 2 is observed for residues 88 and 96 in barnase and for a pocket involving residues 35 and 47 of gene $V$ protein of phage $\mathrm{fl}$ (Kellis et al., 1989; Sandberg \& Terwilliger, 1989). For two sites normally containing charged residues, Asp-80 in kanamycin nucleotidyl transferase (Matsumura et al., 1988b) and Glu-49 in Trp synthase (Yutani et al., 1984, 1987), the slopes vary with $\mathrm{pH}$, reaching a maximum of about 3.8. These latter experiments are more complex insofar as the proteins have significant populations of intermediates (and do not have two-state transitions) and they have an electrostatic component for the transfer. Although it would appear that current results on noncharged residues can be explained largely by different cavity deformabilities (i), nothing rules out the possibility that the other factors (ii and iii) are important. This type of experiment has not yet established whether side-chain freezing is an important component of protein stability.

Which liquid "oil" best characterizes the native core? While there is some evidence that cyclohexane is good (Radzicka \& Wolfenden, 1988), the best correlations of transfer studies with amino acid distributions in proteins appear to involve nonpolar hydrogen-bonding solvents including octanol, ethanol, and dioxane (Fauchere \& Pliska, 1983; Nozaki \& Tanford, 1971; Kyte \& Doolittle, 1982; Rose et al,, 1985a). It may be, therefore, that some fraction of the temperature-independent enthalpy, attributed to van der Waals and hydrogen-bonding interactions, is also present in these transfer experiments and that it contributes differently for different oils.

Finally, I return to the meaning of hydrophobicity. I believe the most useful, common (Tanford, 1980; Ha et al., 1989), and unambiguous meaning of this concept is simply in reference to nonpolar transfers from nonaqueous media into aqueous media: (i) that are strongly disfavored and (ii) whenever there is a large associated increase in heat capacity [definition (2) at the beginning of this section]. It was this remarkable feature of nonpolar solvation that was first identified as unusual (Edsall, 1935; Butler, 1937; Frank \& Evans, 1945 ) and which merits special terminology. Definition (1), on the other hand, needs no special term because it otherwise describes ordinary solution processes. There are two problems with (3), hydrophobicity as water ordering or other molecular models: (i) the molecular mechanism is still not fully understood, and (ii) "water ordering" is an appropriate description of the entropic repulsion of nonpolar solutes near room temperature, but not over a broader temperature range. This would lead to unnecessary hairsplitting: benzene insolubility in water would be referred to as hydrophobic at $25^{\circ} \mathrm{C}$ but not at $100^{\circ} \mathrm{C}$, where it is even more strongly expelled. This meaning is not subject to the Hildebrand objection (1968, 1979); he noted that hydrophobicity is not an enthalpic disaffinity of nonpolar solutes for water but instead is due to a water-water affinity. Also, because definition (2) describes hydrophobicity in terms of the full transfer process, represented by the total free energies in eqs $5-7$ rather than by a particular term in those expressions, it is not subject to difficulties of molecular interpretations (Privalov et al., 1990; Dill, 1990).

\section{(5) What Is MisSING?}

The dominant force of folding is only half the story. Nearly equal in magnitude is a large opposing force. The structures and stabilities of globular proteins result from the balance between driving and opposing forces. Only recently have the main opposing contributions become better understood. That hydrophobicity is not the whole story becomes immediately apparent from certain puzzles. First, Tanford (1962) and Brandts $(1964 a, b)$ showed that hydrophobicity alone would predict protein stability an order of magnitude greater than measured values. They estimated that the free energy of unfolding would be about $100-200 \mathrm{kcal} / \mathrm{mol}$ at $25^{\circ} \mathrm{C}$. This estimate is based on free energies of transfer of hydrophobic amino acids from water into ethanol or dioxane, representative of the folded core of the protein, multiplied by the number of nonpolar residues. However, free energies of unfolding are observed to be only about 5-20 kcal/mol (Pace, 1975; Privalov, 1979; Privalov \& Gill, 1988). This implies that there must be a large force, of magnitude nearly equal to the hydrophobic driving force, which opposes folding. Second, while the temperature dependence of folding was an important clue for hydrophobicity, Tanford (1962) suggested that it also posed a paradox. If nonpolar components associate more strongly as the temperature is increased, then proteins should fold more tightly with increasing temperature. Just the opposite is observed above room temperature: increasing temperature unfolds proteins. Proteins are typically thermally unfolded in the range of $50-100^{\circ} \mathrm{C}$; the free energies of transfer of hydrocarbons into water have a maximum extrapolated to be in the range of $130-160^{\circ} \mathrm{C}$. Third, if hydrogen bonding is not a dominant force of folding, then what is the origin of the considerable amounts of internal architecture, of secondary and tertiary structures, in proteins? Through what forces do the amino acid sequences so uniquely determine the native structure? The hydrophobic effect would seem to be too nonspecific and an unnatural candidate as the origin of helices and sheets.

Fourth, the pressure dependence of protein stability does not resemble that of model hydrophobic compounds (Brandts et al., 1970; Zipp \& Kauzmann, 1973; Kauzmann, 1987). For example, the partial molar volume of methane decreases from 60 to $37.3 \mathrm{~mL} / \mathrm{mol}$ upon transfer from hexane to water (Masterson, 1954). The decreased volume arises because water molecules pack more efficiently surrounding a nonpolar solute molecule than in its absence. Since the unfolding of a protein leads to increased nonpolar exposure to water, then these model studies would suggest that the partial molar volume of a protein should decrease considerably upon unfolding due to similar contraction of solvating water molecules (by about $20 \mathrm{~mL} / \mathrm{mol}$ multiplied by a number in the range of 10-40, representing the total hydrophobic exposure upon unfolding). While the volume change of protein unfolding is indeed generally observed to be negative, it is only in the range of -30 to $-300 \mathrm{~mL} / \mathrm{mol}$ (about $0.5 \%$ of the total volume) (Brandts et al., 1970; Zipp \& Kauzmann, 1973; Edelhoch \& Osborne, 1976; Richards, 1977), which is somewhat smaller than the methane model would suggest. Brandts et al. (1970) suggested that other simple factors would not account for this discrepancy; for example, if unfolding leads to exposure of charged groups, then the volume change upon unfolding would be predicted to be even more negative, increasing the discrepancy. There is an additional problem. For model compounds, increasing the pressure leads to more normal water solvation, so the volume change of transfer to water diminishes and ultimately becomes positive at about 1500-2000 atm. For proteins, on the other hand, the negative volume of unfolding does not change much with pressure. Two simple explanations, however, can account for these discrepancies between the 
model transfer experiments and protein unfolding. First, methane is a poor model amino acid. Better models include alcohols, ketones, and amides, which can form hydrogen bonds; these model compounds have much smaller negative volumes of transfer to water, more closely predictive of the protein experiments (Friedman \& Scheraga, 1965; Hvidt, 1975). Second, the pressure dependence is at least qualitatively accounted for by recognizing (i) that model nonpolar solutes in water are less compressible than in the pure liquid (Brandts et al., 1970) and (ii) that the folded state of a protein is much less compressible than the reference liquid hydrocarbon to which it is generally compared. A folded protein is typically 10-fold less compressible than organic liquids (or about half as compressible as ice!) (Kundrot \& Richards, 1987; Gavish et al., 1983; Eden et al., 1982; Klapper, 1971; Fahey et al., 1969). Due to (i), the volume change in small-molecule transfers should diminish with increasing pressure; due to (ii), the volume change in protein folding should diminish much less with increasing pressure than in the small-molecule reference experiment. Therefore, it is important to recognize that a protein is not just a sum of transfers of small-molecule model side chains. Proteins are polymers. It is described below how the chainlike nature of proteins and the resultant conformational freedom lead to a strong force that opposes folding.

\section{(6) Principle Opposing Force Is Entropic}

Since the 1930s it has been known that the main force opposing protein folding is entropic. Northrop (1932) was the first to observe a sharp thermal denaturation transition; the equilibrium constant depends strongly on temperature. Only more recently has the molecular basis for the opposing entropy become clear. Just as there are translational, rotational, and vibrational entropies of small molecules, depending on the relevant degrees of freedom, likewise there are different possible molecular origins of the entropy gain upon protein unfolding. For example, Mirsky and Pauling (1936) suggested that folding would be opposed by an entropy arising from the proper mating of specific ion pairs. As another example, the helix-coil transition theories (Schellman, 1958a; Zimm \& Bragg, 1959; Poland \& Scheraga, 1970) showed that local degrees of freedom could be an important source of entropy opposing helix formation.

However, it has long been known that polymers are also subject to another type of configurational entropy, one which is nonlocal (Flory, 1953; deGennes, 1979). It arises from "excluded volume", the impossibility that two chain segments can simultaneously occupy the same volume of space. A chain can occupy a large volume of space in any of a large number of different configurations. However, there are relatively few ways the chain can configure if it is constrained to occupy a small volume of space, simply due to severe steric constraints.

Excluded volume (steric constraints) will play a role in any process that involves a change in the spatial density of polymer segments. These include solution thermodynamic properties of chain molecules, particularly their dependence on concentration, including solubilization and phase behavior, colligative properties, expansion and shrinkage, and virial coefficients and their temperature dependences (Flory, 1953; Munk, 1990). Models for predicting these effects originated with the Flory-Huggins theory (Flory, 1953); others are based on scaling (deGennes, 1979) and renormalization group methods (Freed, 1987).

How can a local entropy be distinguished from a nonlocal entropy? By definition, the entropy is $S / N k=-\sum_{i=1}^{s} p_{i} \ln$ $p_{i}$, where the probabilities $p_{i}$ describe the distribution of all states, $i=1,2,3, \ldots, s$, accessible to a system, and $N$ is the number of particles. The issue of local vs nonlocal is the question of what degrees of freedom change in the process of interest. Local entropies arise from the energies responsible for the conformations of connected residues:

$$
p_{i}=z^{-1} \exp \left[-\epsilon\left(\phi_{i}, \psi_{i}, \chi_{i}, \chi_{i+1}\right) / k T\right]
$$

where $z$ is the partition function and $\epsilon$ is the energy of the dipeptide (or tripeptide, etc.) as a function of $\phi, \psi$, and $\chi$ angles. These resemble vibrational or internal rotational entropies in small molecules; their contribution to stability is probably small (Karplus et al., 1987). Local entropies are independent of global properties of the chain such as the radius of gyration, or internal/external distributions of nonpolar/polar residues. Local entropies underlie helix/coil processes. There is evidence that changes in local entropies can affect the stabilities of globular proteins. For example, Matthews et al. (1987) have shown that replacement of a glycine for a proline in T4 lysozyme decreases protein stability by increasing the local conformational freedom of one peptide bond.

On the other hand, nonlocal entropies depend on the relative numbers of chain configurations, $N(\rho)$, as a function of the chain segment density, $\rho$ (i.e., the number of monomers divided by the volume occupied by the chain):

$$
p(\rho)=\frac{N(\rho)}{\int N(\rho) \mathrm{d} \rho}
$$

Because the folding of a protein involves collapse of the chain from a large volume (the denatured state) to a small volume (the native state), it must lose a considerable amount of this nonlocal entropy in the process. Native states of globular proteins are extremely compact (Klapper, 1971; Richards, 1977). They have as little free volume as smallmolecule crystals; they have compressibilities closer to those of glasses than to those of liquids; and their configurational freedom is as restricted as in glasses and crystals of polymers, as evidenced by the existence of virtually unique native configurations of proteins.

One test of the importance of nonlocal entropies in protein stability involves cross-linking the chain. Introducing a cross-link reduces the number of conformations accessible to the unfolded state, making it relatively less favorable, and in that way making the folded state relatively more stable (Kauzmann, 1959; Poland \& Scheraga, 1965; Chan \& Dill, 1989a, 1990a; Pace et al., 1988; Matsumura et al., 1989). It appears that the most effective way to stabilize a protein at present, with a single amino acid change, is to add a cross-link. Increases of 29 and $25^{\circ} \mathrm{C}$ in denaturation temperature have been achieved in hen lysozyme and ribonuclease (Alber, $1989 \mathrm{~b}$ ). For the same reasons, a protein should also be stabilized if it is constrained to be adjacent to an inert surface or contained within a small pore (Dill \& Alonso, 1988). Similarly, the restriction of configurations of the unfolded chain may also account for the observation that carbohydrates or other chains appended to proteins appear to stabilize them (Burteau et al., 1989).

\section{(7) Modeling Protein Stability}

More quantitative insights into the forces of protein folding can be obtained through theoretical models and experimental tests of them. In principle, if the hydrophobic interaction and conformational entropy are the dominant contributions, then the free energy of folding can simply by calculated as (i) the difference in nonpolar exposure in native and denatured states, multiplied by the free energy of transferring nonpolar surface, and (ii) the difference in conformational entropies. The free 
energy of folding has been calculated in various studies, on the basis of several of the following simplifying assumptions: (i) in the native state the hydrophobic residues are fully buried in a nonpolar core and (ii) in the unfolded state the hydrophobic residues are fully exposed to solvent. The hydrophobic contributions would then be the sum of the free energies of transfer of the nonpolar residues. If in addition (i) there is only one configuration in the folded state, (ii) the denatured state is an ideal random flight [as in a "theta" solvent (Flory, 1953)], and (iii) the entropy is local, then the difference in configurational entropies will be simply $n k \ln z$, where $n$ is the number of rotational bonds and $z$ is the number of accessible conformations per dipeptide bond. These approaches have not succeeded, perhaps for the following reasons. First, native states have nonpolar exposure. Methods now exist, however (Lee \& Richards, 1971; Eisenberg \& McLachlan, 1986), to determine the detailed surface exposure and the associated free energies, if the native structure is known. Second, full exposure of the unfolded state is a poor approximation. The ensemble of denatured configurations represents a complex polymeric state, with the following properties. (1) It is often dense, being only 1.3-2-fold greater in volume than the native state, rather than 10-100-fold as in the theta state (Privalov et al., 1986; Ptitsyn, 1987; Goto et al., 1990). (2) The net change in surface exposure to water upon unfolding can be as little as $14 \%$ of the maximum possible (Tanford, 1968; Tanford, 1970; Ahmad \& Bigelow, 1986; Schrier \& Schrier, 1976; M. Hurle, private communication). (3) The radius, and therefore the free energy, of denatured configurations depends on the composition and length of the chain (Tanford, 1968; Goto et al. 1990; Shortle et al., 1988) and on external thermodynamic conditions, including temperature, $\mathrm{pH}$, salt, and denaturants (Privalov et al., 1986). (4) In some cases there are distinctly identifiable different nonnative states and configurational transitions between them (Evans et al., 1987; Goto \& Fink, 1989, 1990; Goto et al., 1990; Shortle \& Meeker, 1989). (5) Some nonnative states have much secondary structure (Ptitsyn, 1987; Baum et al., 1989; Shortle \& Meeker, 1989; Shortle et al., 1988). This is not a class of configurations that can be represented as fully exposed and independent of the solvent and thermodynamic conditions. For these reasons it is also clear that the configurational entropy of folding cannot be due principally to local factors and is not a constant independent of chain and solvent properties. Because the unfolded state contributes to stability on the same footing as does the folded state, satisfactory predictions of stability will require satisfactory models of the unfolded states.

Arguably, the simplest model for polymer chain collapse that treats the chain conformations more realistically is that of homopolymers in poor solvents. The nonlocal conformational entropy favors the open configurations, but when the solvent becomes poor (incompatible), the polymer collapses because polymer-polymer contacts are more favorable than polymer-solvent contacts (Lifschitz, 1968; deGennes, 1975; Post \& Zimm, 1979; Sanchez, 1979).

The collapse of a heteropolymer such as a protein is significantly different than that of a homopolymer. In the process of collapse to the folded state, the ensemble of configurations of the molecule must (i) decrease in radius to native compactness and (ii) configure so as to bury much nonpolar surface area (see Figure 7). Hence, there are two degrees of freedom: the radius (or segment density) and the "ordering", the degree of segregation of the nonpolar residues into a core. In contrast, for homopolymers the only degree of freedom is the density. If the free energy is known as a function of these two degrees

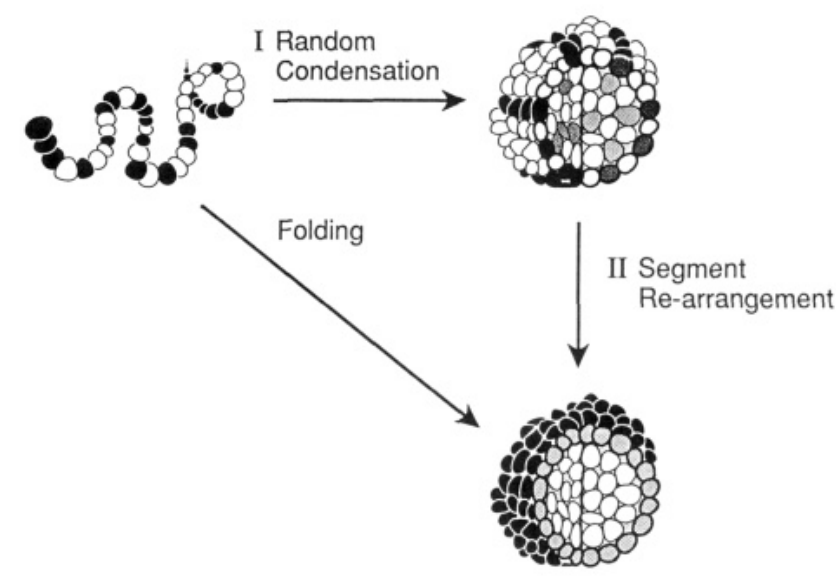

FIGURE 7: Protein folding involves (I) an increase in compactness of the chain and (II) a reconfiguration of nonpolar residues into the core and polar residues to the surface. The collapse of homopolymers only involves (I).

of freedom, then the state of minimum free energy can be found. Theory has recently been developed on this basis for the collapse of heteropolymers (Dill, 1985; Dill et al., 1989). The protein is represented as a chain of bead-like monomers connected by rotatable bonds. Two minima are generally found in this model. One minimum identifies a compact state with nonpolar core, the folded state, and the other minimum identifies a less compact ensemble (with no nonpolar core), the distribution of which changes with solution conditions; this represents the unfolded ensemble. Because there is a free energy barrier between these two states, the model predicts two-state behavior, wherein intermediate states are less populated than native or denatured states. This agrees with experiments, which show that many small single-domain proteins populate predominantly two states (Lumry et al., 1966; Privalov \& Kechinashvili, 1974; Privalov, 1979). This approach addresses the problems above: the nature of the unfolded state and its nonpolar exposure are predicted from the balance of forces rather than assumed, and the conformational entropy difference between native and denatured states is calculated as a function of the chain and solution properties. Thus the theory simply provides a procedure for enumerating (i) the relative amount of nonpolar surface buried in the folded and unfolded states (some nonpolar surface is found to be exposed in the folded state, and some is buried in the unfolded state) and (ii) the number of configurations accessible in the folded and unfolded states. Three approximations are used for this counting (Dill, 1985; Dill et al., 1989). First, the BraggWilliams mean-field approximation (Hill, 1960) is used to count nonpolar contacts by assuming they are uniformly distributed within the chain volume. Second, the Flory (1953) mean-field approximation is used to calculate how the number of conformations diminishes with density due to excluded volume; it too assumes segments are uniformly distributed. Third, only the effects of composition are taken into account (the number of nonpolar residues, and not their sequence along the chain): this is the random copolymer approximation. On this basis, the free energy of folding depends on (i) the chain length, (ii) the number of nonpolar residues, and (iii) the free energy of transferring an amino acid from water to a suitable "protein-like" medium.

The general predictions of the model are at least qualitatively consistent with known protein behavior. For example, the theory predicts that proteins should undergo a (maximally cooperative) first-order transition from the denatured to native state as the solvent becomes poor (Dill, 1985; Dill et al., 1989). 


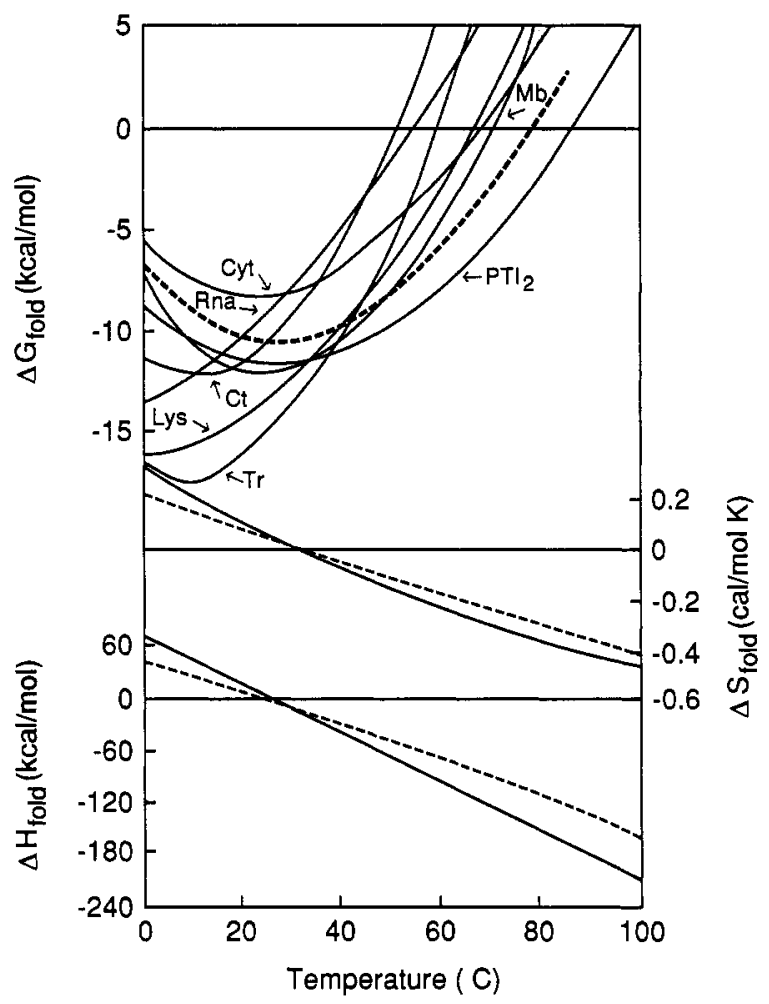

FIGURE 8: Thermal stabilities of proteins. Experimental data for free energies, enthalpies, and entropies of folding taken from Privalov (1979) and Privalov and Kechinashvili (1974) (-). Theoretical predictions are from Dill et al. (1989) $(--)$.

If there are too few hydrophobic residues, or if the chain is too short (less than several tens of residues), the molecule will not fold. The hydrophobic free energy is predicted to be about $60 \mathrm{kcal} / \mathrm{mol}$ of protein at $25^{\circ} \mathrm{C}$ for typical small proteins (Dill et al., 1989). The opposing configurational free energy is predicted to be of nearly the same magnitude as the hydrophobic force: about $50 \mathrm{kcal} / \mathrm{mol}$ at $25^{\circ} \mathrm{C}$. This configurational entropy is large because there are tens of orders of magnitude fewer configurations in the folded than in the unfolded states (Chan \& Dill, 1990a; Dill, 1985). Thus, the net stability of the protein should be a small difference of these two large free energies. This also resolves a related problem. The number of configurations of the unfolded protein is enormous. On that basis, it has been suggested that a protein would require many ages of the universe to find the native structure by random search, and thus that it could not attain its thermodynamic state of lowest free energy. This theory, on the other hand, predicts that simple collapse will reduce the configurational space enormously, implying that there is no inconsistency with the observation that proteins do attain their states of minimum free energy (Dill, 1985).

The theory also predicts the nature of thermal and solvent stabilities of proteins, in good agreement with experiments. To predict thermal stability, the temperature dependence of the hydrophobic interaction must be put into the model; this is taken (Dill et al., 1989) from the nonpolar small-molecule transfer experiments of Nozaki and Tanford (1971) and Gill and Wadsö (1976). The theory then predicts the existence of two first-order transitions vs temperature. One, a "cold" denaturation, occurs at low temperatures because the dominant temperature dependence of folding at those temperatures is due to the weakening of the hydrophobic interaction with decreasing temperature. Cold denaturation has been observed or has been predicted from extrapolations of experiments in several systems (Christensen, 1952; Hawley, 1971; Privalov et al., 1986; Chen \& Schellman, 1989; Brandts, 1964a; Pace
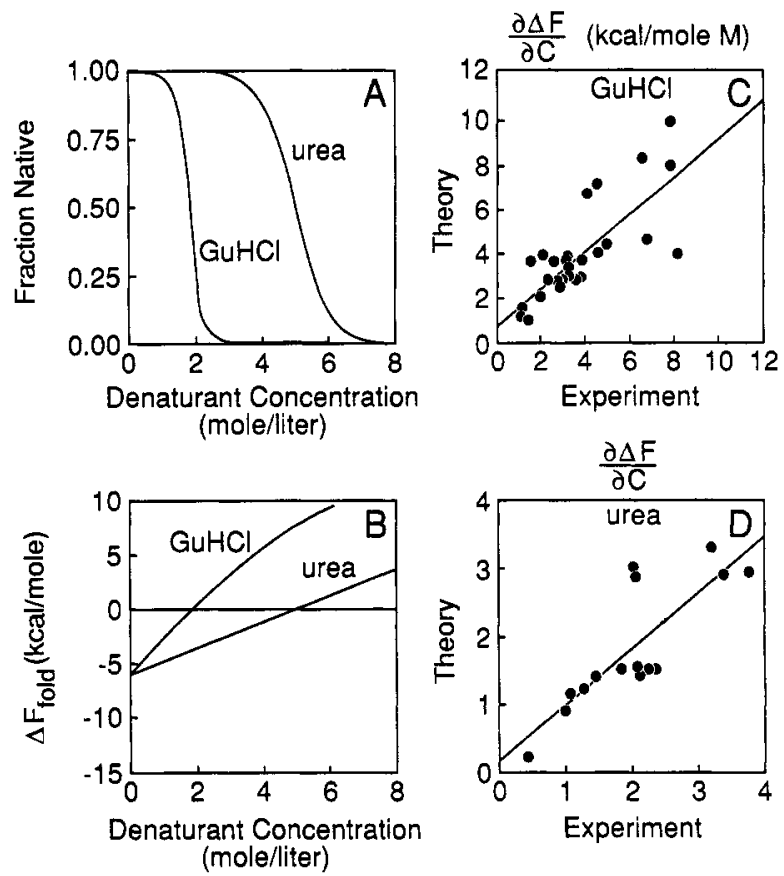

FIGURE 9: Comparison of theory and experiments for denaturation of proteins by guanidine hydrochloride and urea (Alonso and Dill, 1990). (A) Theoretical denaturation curve. (B) Free energy vs denaturant has approximately constant slope for small ranges of denaturant. (C and D) Comparison of theoretical and experimental slopes, $\partial \Delta F_{\text {fold }} / \partial c$, at the denaturation midpoint.

\& Tanford, 1968; Privalov, 1979; Privalov \& Gill, 1988; Griko et al., 1988). The other more familiar thermal denaturation at higher temperatures occurs because the gain in conformational freedom of the chain is more advantageous than the gain of interactions among nonpolar contacts. Comparison of the theoretical and experimental free energies, enthalpies, and entropies of folding is shown in Figure 8.

The theory has also been applied to the prediction of protein stabilities in denaturing solvents, such as urea and guanidine hydrochloride, and in stabilizing solvents (Alonso \& Dill, 1990). In general the model can be applied to predicting stability as a function of any external parameter, $x$, provided that the oil/water partition coefficient for the representative elementary amino acid transfer is known as a function of $x$. In the case above, $x$ is temperature; in this case, $x$ is the concentration of urea or guanidine in the aqueous solvent. With use of the small-molecule transfer data of Nozaki and Tanford (1970) for this purpose, the resultant protein stability theory predictions are shown in Figure 9, along with experimental results. The theory predicts that the free energy of folding should be a linear function of urea concentration or a slightly curved function of guanidine concentration. These denaturants are predicted to cause unfolding by solvating nonpolar groups better than water does. Similarly, proteins should be stabilized by additives that are worse at solvating nonpolar groups than water is. Stabilizers include a wide range of sugars (Lee \& Timasheff, 1981; Arakawa \& Timasheff, 1982; Back et al., 1979), glycerol (Gekko \& Timasheff, 1981), and polyols, poly (ethylene glycol), and some salts (Back et al., 1979; Arakawa et al., 1990).

\section{(8) Why Do Proteins Have Internal Architecture?}

Globular proteins have internal organization, comprised of a combination of "irregular" structures and "regular" structures such as helices and sheets. This internal organization is uniquely specified by the amino acid sequence. This organization and its uniqueness would appear to be difficult to 
reconcile with the picture of the nonspecific dominant forces described in the preceding sections. Secondary structures are hydrogen bonded. Therefore, hydrogen bonds must naturally play a significant role in determining internal architecture. Yet according to the arguments above, hydrogen bonding, although prevalent in folded proteins, is a weak driving force. Moreover, hydrogen bonding taken alone cannot readily explain sequence specificity: how the sequence encodes only one native conformation. Most intrachain hydrogen bonds in globular proteins are among backbone $\mathrm{C}=\mathrm{O}$ and $\mathrm{NH}$ groups rather than among side chains (Baker \& Hubbard, 1984). From the data base of protein crystal structures, Baker and Hubbard observed that, of all the intrachain hydrogen bonds to $\mathrm{C}=\mathrm{O}$ groups, $81.3 \%$ are with backbone NH groups and $18.7 \%$ are with side chains. Of all the intrachain hydrogen bonds to $\mathrm{NH}$ groups, $86.2 \%$ are with backbone $\mathrm{C}=\mathrm{O}$ groups and $13.8 \%$ are with side chains. However, sequence specificity must arise from differences in side-chain properties, not from differences in backbone properties such as peptide hydrogen bonding. Any one amino acid will have essentially the same backbone interactions as any other. Therefore, if hydrogen bonding were dominant, the native structure of a protein should be essentially independent of the amino acid sequence, and native structures should be regular and periodic, either purely helix or purely sheet. Similarly, to the extent that backbone van der Waals interactions favor folding, they also are probably not very selective for one compact conformation relative to others.

Could it be that there is so much secondary structure in proteins because irregular conformations cannot form hydrogen bonds as well as helices and sheets can? The current limited evidence does not support this view. That the many irregular conformations of proteins can form good intrachain hydrogen bonds is clear from the study of Baker and Hubbard (1984). Of all the hydrogen bonds to $\mathrm{C}=\mathrm{O}$ groups, $8.9 \%$ are in $\beta$ structures, $24.6 \%$ are in helices, and $5.3 \%$ are in turns. In comparison, a minimum of $18.3 \%$ of all hydrogen bonds to $\mathrm{C}=\mathrm{O}$ groups are in "irregular" structures; i.e., they are intrachain (not bonded to water) and not in secondary structures. This is a minium because the Baker and Hubbard study does not itemize bonds to water and in secondary structures as mutually exclusive categories, so only this lower bound can be obtained from their study. Similarly for NH groups, $38.3 \%$ are hydrogen bonded in helices, $13.6 \%$ in $\beta$-structures, and $9 \%$ in turns. A minimum of $17.9 \%$ of all hydrogen bonds to $\mathrm{NH}$ groups are in irregular structures. Hence, hydrogen bonds in irregular structures are not significantly less common than those in helices and sheets. Combined with the observation that $11 \%$ of all $\mathrm{C}=\mathrm{O}$ groups and $12 \%$ of all $\mathrm{NH}$ groups have no hydrogen bonds (Baker \& Hubbard, 1984), this suggests that hydrogen-bonding requirements do not severely constrain the conformations accessible to the chain.

Indeed, the earliest expectations (Wu, 1929; Pauling et al., 1951; Pauling \& Corey, 1951a-d; Kendrew et al., 1958; Kendrew, 1961) were that internal architecture in globular proteins would be regular and periodic as in crystals, presumably with all residues in helices or sheets. For example, Pauling et al. (1951) and Pauling and Corey (1951a-d) specifically sought types of structure in which every amino acid was "equivalent", i.e., interchangeable and not dependent on the sequence. Therefore, ever since the appearance of the first known structures of globular proteins (Kendrew et al., 1958), the central problem of protein architecture has not been to explain why proteins have so much regular structure. It has been just the opposite: to explain why there is so much irregular structure, and how the sequence uniquely encodes them both. About $53 \%$ of all residues in globular proteins are in irregular structures (Kabsch \& Sander, 1983; Kneller et al., 1990). Is protein architecture then a consequence of the smaller interactions, including-side chain hydrogen bonding? In the following section, I suggest that sequence-specific internal architecture in globular proteins does not arise principally from these smaller forces but from the dominant forces.

Recent exhaustive simulations of all the possible conformations of chains have shown that protein-like internal architecture is simply a natural consequence of steric constraints in compact polymers (Chan \& Dill, 1989a,b, 1990a,b). Any flexible polymer, when made to be compact by any driving force, will have much internal architecture composed of helices and sheets. For proteins this driving force is presumably the hydrophobic interaction. There are simply very few possible ways to configure a compact chain, and most of them involve helices and sheets. The existence of internal organization is due to the physical impossibility of steric violation which alternative configurations would require. In other words, consider a pearl necklace. Squeeze it into a ball. One might expect that an ensemble of these compact necklaces would be highly disordered. This is not the case, however; it will have approximately the same distribution of internal architectures as are observed in globular proteins, comprised of helices, sheets, and irregular structures. The evidence for this is the following.

Polymer simulations and theory show that if a chain molecule is to form a single self-contact, it will prefer one that forms the smallest possible loop (Jacobson \& Stockmayer, 1950; Poland \& Scheraga, 1965; Chan \& Dill, 1989a, 1990a). A chain with a local (small) loop has more remaining accessible conformations (and thus greater entropy) than a chain with a nonlocal (large) loop. [Theory and experiment also show that "stiffness", i.e., intrinsic propensities, can further favor or disfavor tight loops (Flory, 1969; Semlyen, 1976; Zhang \& Snyder, 1989).] Now consider configurations that have two self-contacts; these provide the most basic description of elements of secondary structure. A most interesting result from the theory and simulations is that if a chain is to form two self-contacts, it will again prefer to form a small loop and to form the second contact as close as possible in sequence to the first, simply because these, among all possible two-contact configurations, have the greatest entropy (Chan \& Dill, 1989a, 1990a). The only two ways a chain molecule can form such a loop pair are either as a helix or as the beginning of an antiparallel sheet. Hence given only the conformational freedom and steric restriction in flexible chains, there is a tendency to form helices and sheets, even in the absence of other forces, and even for chain conformations of relatively large radius of gyration. These results are obtained for chains configured in two or three dimensions, on different types of lattices, and by alternative path integral methods and therefore do not appear to be an artifact of the theoretical methods used in these predictions.

Exhaustive simulations further show that as a chain becomes increasingly compact, it develops a considerable amount of secondary structure (Chan \& Dill, 1989b) (see Figure 10). Consistent with this result, protein-like organization in compact chains has been observed by Monte Carlo methods for other lattices and chain lengths (Sikorski \& Skolnick, 1989; Skolnick et al., 1988, 1989).

The internal distributions of secondary structures obtained in exhaustive simulations of all possible compact conformations (Chan \& Dill, 1990b) are in good agreement with the distributions that Kabsch and Sander (1983) have observed in 

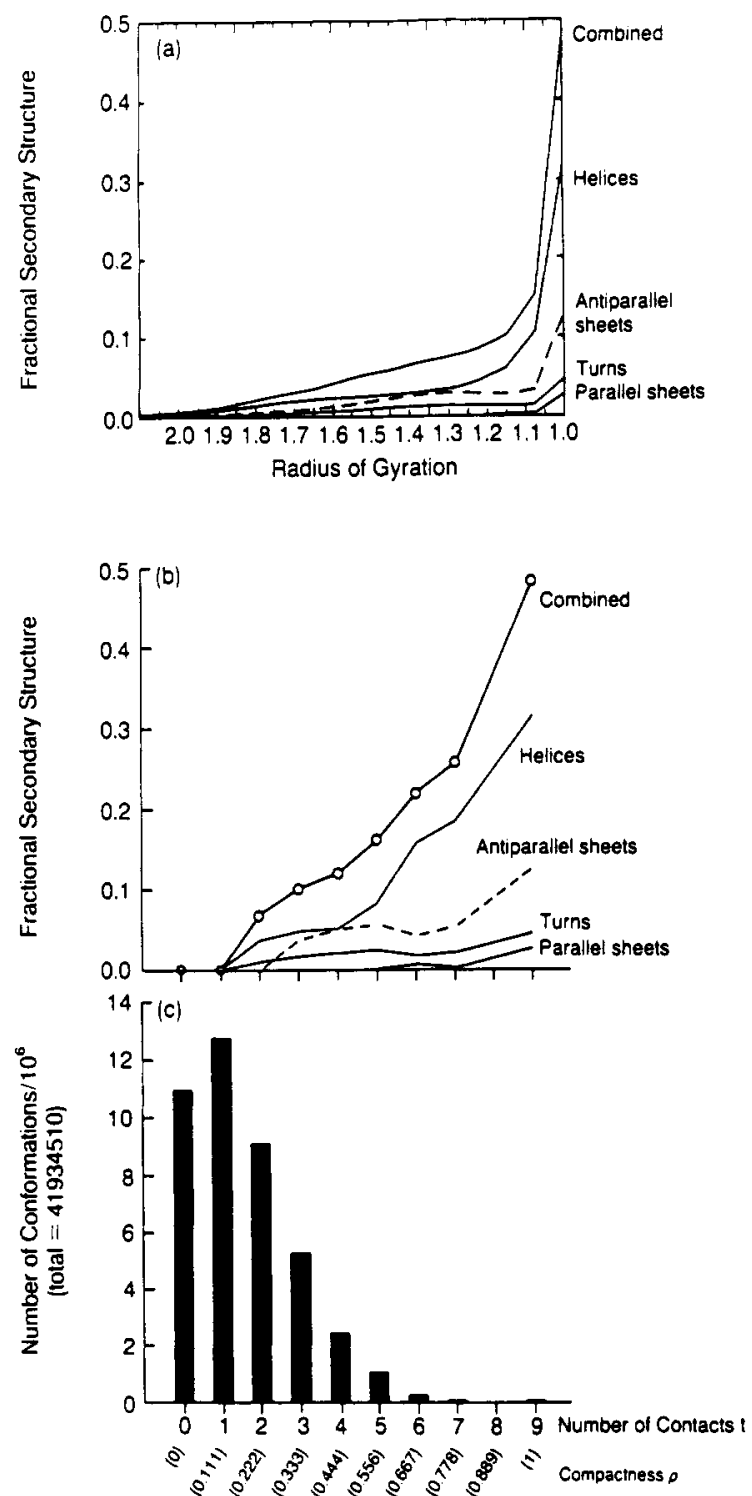

FIGURE 10: Increasing compactness of a chain molecule leads to the formation of secondary structure. With increasing compactness (number of intrachain contacts), exhaustive simulations of all possible configurations of short chains on lattices show that (Chan \& Dill, $1989 \mathrm{~b}, 1990 \mathrm{~b}$ ) (c) the number of accessible configurations diminishes rapidly and (b) and the amount of secondary structure increases sharply. (a) Same results as (b), except plotted against radius of gyration instead of compactness.

the data base of protein crystal structures (see Figure 11). For example, theory and experiment agree that the most common helices and sheets will be the shortest ones; that the shortest antiparallel sheets are 3-4-fold more common than the shortest helices, which are about as common as the shortest parallel sheets; and that, among long secondary structures, in decreasing prevalence should be helices, antiparallel sheets, and then parallel sheets. It is interesting then to turn this argument around. Since the known protein architectures are distributed in the same way as in the complete ensemble of all compact conformations, it suggests that the currently known proteins are a reasonably representative sample of all the forms of internal structure that proteins could adopt [see also Finkelstein and Ptitsyn (1987)].

These results provide an explanation for certain aspects of protein structure that have otherwise been puzzling. First, they show how the dominant forces due to hydrophobicity and steric constraints give rise to internal architectures. They provide a single framework for comprehending the coexistence of all
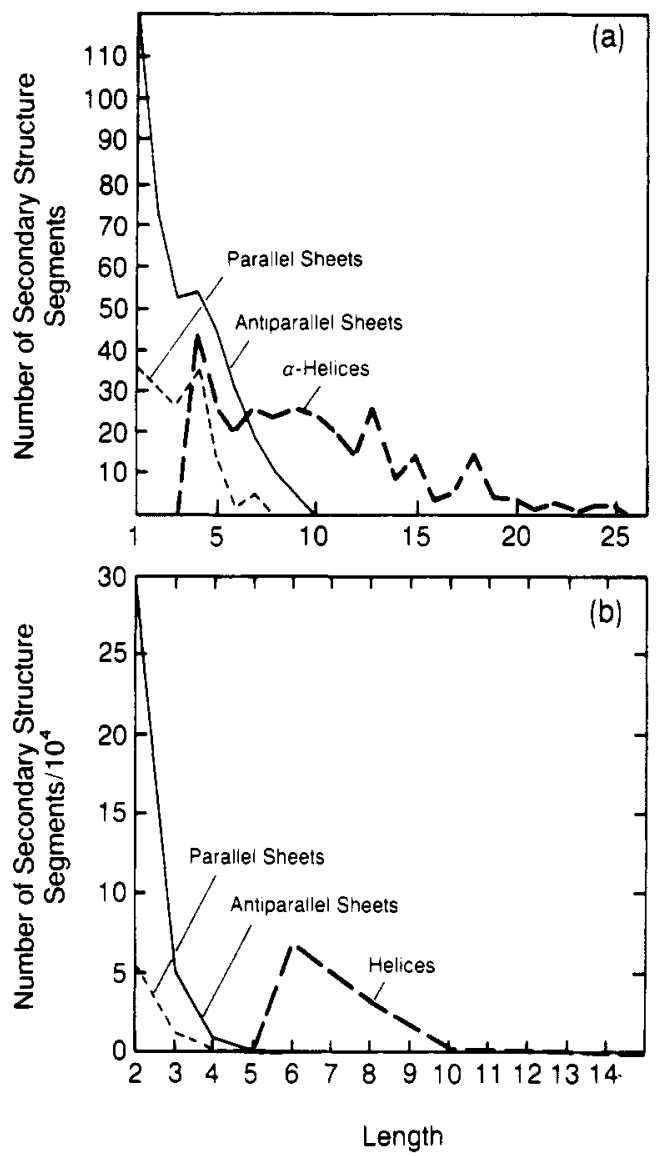

FIGURE 11: Comparison of data base studies of Kabsch and Sander (1983) (a), of the internal distributions of lengths of helices and sheets in globular proteins, with (b) exhaustive lattice simulations of the full compact ensemble (Chan \& Dill, 1990b).

the types of internal protein structure, regular and irregular, helices and sheets. Past hypotheses have tended to address a single type of architecture, often principally helices, in which the focus is on factors that are local in the sequence. The present results suggest instead that architectures arise principally from nonlocal factors. That is, "tertiary" forces drive secondary structures rather than "secondary" forces driving tertiary structures. It follows that helices and sheets in globular proteins are only secondarily a consequence of hydrogen bonding. In this regard, it is interesting that of the 176 known crystal structures of different synthetic polymers that are considered to be reliable, 49 are planar zigzags, and 79 are helices of 22 different types (Tadokoro, 1979). Many different crystalline polymers have helical pitches nearly the same as that of the $\alpha$-helix, between three and four monomers per turn, including polybutadiene, polypropylene, polyvinylnaphthalene, polybutene, and even fibrous sulfur, none of which form hydrogen bonds (Takodoro, 1979). This is not to say that local factors and hydrogen bonds are unimportant. This is only to say that local factors and hydrogen bonding probably contribute to making energetic "decisions" only within an already highly restricted ensemble, one in which the chain will be forced (by the hydrophobic drive to compactness and by the highly selective steric forces) to have considerable stretches of $(i, i+3)$ self-contacts, for example. In that regard, what dictates that these will be specifically $\alpha$-helices, with 3.6 residues per turn, instead of any of dozens of other types of closely related helices will be local forces uniquely determined by amino acids as monomers.

One aspect of internal architecture that is not a consequence of packing forces alone is the spatial distribution of turns. Turns are observed to occur largely at the surfaces of proteins 
(Kuntz, 1972). This does not arise from packing constraints (Chan \& Dill, 1989b). Rather it appears to be a consequence of the polar nature of turn residues (Rose et al., 1985b). Since the middle residues of turns are geometrically unable to form intrachain hydrogen bonds, then their hydrogen bonding needs can best be met by interacting with water at protein surfaces.

A second puzzle has been why some denatured states of proteins have secondary structure. According to these simulations, in the absence of other interactions, the amount of secondary structure simply depends on the radius of gyration of the chain (Chan \& Dill, 1989b, 1990b) (see Figure 10). Therefore, proteins that are denatured only weakly, and thus have small radii, should have some secondary structure. This may account for secondary structures in "molten globule" and other compact denatured states of proteins, with radii only slightly larger than those of native molecules (Ptitsyn, 1987; Goto \& Fink, 1989, 1990; Shortle \& Meeker, 1989; Kuwajima, 1989; Brems \& Havel, 1989; Baum et al., 1989).

Because internal architecture should therefore arise in any compact polymer, it should be possible to design other copolymers, not necessarily comprised of amino acids, which can be driven to compactness in poor solvents and which should then have protein-like architectures. Consistent with this prediction, Rao et al. (1974) have shown that in a solution containing a large number of different sequences of amino acid copolymers roughly half the molecules are highly compact and there is $46 \%$ helix observed by circular dichroism. This implies that a large fraction of all possible sequences is capable of coding for large amounts of helix.

\section{(9) Why Is the Native Structure Unique?}

A most unusual feature of globular proteins, relative to any other type of isolated polymer molecule, is that they can be found in only a single conformation, the native structure. ("Single" here means that the chain path is identifiable at relatively high resolution as an average over the ensemble of small fluctuations.) What forces, encoded in the amino acid sequence, cause this remarkable uniqueness? As noted above, there is considerable elimination of configurational possibilities simply due to the compactness. Nevertheless, even for compact chain molecules, there are still many accessible conformations; the number of maximally compact conformations increases exponentially with chain length (Chan \& Dill, 1989b; Dill, 1985). Within this ensemble of physically accessible compact conformations, all the various types of interactions could play a role, including hydrogen bonding, intrinsic propensities, ion pairing, and hydrophobicity, in causing some compact conformations to be of higher free energy and others to be of lower free energy.

Is there a single one among these types of interaction, by virtue of its encoding within the sequence, that "picks out" the one native structure? The most significant further restrictor of conformational space may be the hydrophobic interaction. By use of a simple model of short self-avoiding flexible chains on lattices, in which the only energetic feature of the sequence is the hydrophobic interaction, every conformation has been explored by exhaustive search in order to determine the native state(s), those at the global minimum of free energy (Lau \& Dill, 1989; Chan and Dill, unpublished results). This has been done for many different sequences. In this model, residues are either hydrophobic $(\mathrm{H})$ or polar $(\mathrm{P})$. The free energy of any chain conformation is determined simply by the number of $\mathrm{HH}$ topological contacts (topological contacts are defined in Figure 1). Therefore, "native" conformations are those with the maximum number of such $\mathrm{HH}$ contacts. How many native structures does any sequence have? Figure 13 shows the
For The Sequence: H P PH PHHPHPHHH 1234567891011121314

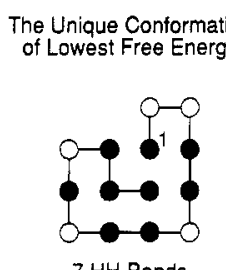
Other Compact Conformations of Higher Free Energy (Fewer HH Bonds)

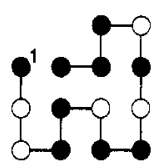

$4 \mathrm{HH}$ Bonds

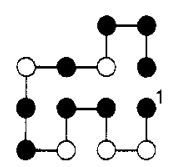

$4 \mathrm{HH}$ Bonds

FIGURE 12: Exhaustive lattice simulations of short chains show that any given sequence has many different compact conformations (such as the two on the right) but often only one native state, in which the maximum number of nonpolar contacts is formed. Nonpolar residues $(\mathrm{H}, \bullet)$; polar residues $(\mathrm{P}, \mathrm{O})$.

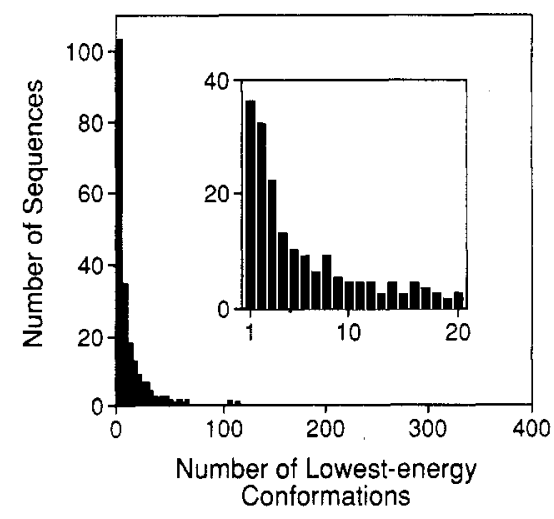

FIGURE 13: How many native structures does a given sequence have? This histogram derives from a 2D lattice model of self-avoiding flexible chains (of length $=24$ monomers) subject only to hydrophobic in. teractions (Lau \& Dill, 1990). Exhaustive search permits determination of the structure(s) of global minimum in free energy among the compact ensemble. These native structures are then found for many different sequences. The inset shows finer detail. This decreasing function implies that more sequences have only one native structure than have two, three, etc. Thus for a typical sequence, there are very few configurations that have the maximum possible number of nonpolar contacts.

distribution. The most surprising result is that this is a strongly decreasing function; far more sequences can configure into only one native structure than can configure into 10 or 100 native structures. Hence according to this simulation, for most folding sequences there are exceedingly few ways a chain can configure to form the maximum possible number of $\mathrm{HH}$ contacts. One example is shown in Figure 12. This suggests that hydrophobicity is strongly selective and singles out only a very small number of candidate native structures from the compact ensemble. Other evidence also supports this view. Hydrophobicity patterns appear to be more predictive of conformational families than other types of interactions (Sweet \& Eisenberg, 1983; Bashford et al., 1987; Bowie et al., 1990). In addition, Covell and Jernigan (1990) have exhaustively explored all conformations of lattice chains confined within known protein shapes using 3D lattices, weighting them using the free energies of Miyazawa and Jernigan (1985), and have compared them with known protein structures. They found the native conformation to be within the best $1.8 \%$. Hence within their shape-restricted ensemble, the transfer free energies, whatever their molecular bases in hydrophobicities, hydrogen bonding, van der Waals, and other interactions, considerably restrict the possible native structures. Thus, the nonlocal forces encoded within the sequence, rather than the local factors involving connected residues, would appear to be largely responsible for the uniqueness of the native structure.

Whatever role hydrophobicity may play in reducing the ensemble to a small set of possibilities, it is also clear that 


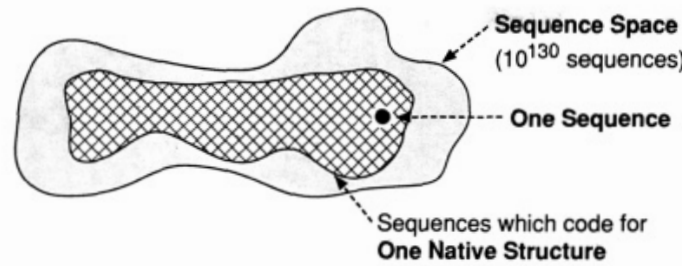

FIGURE 14: For a protein of 100 residues, there are $20^{100} \cong 10^{130}$ different sequences. This is the sequence space. A given protein (such as ribonuclease) has only one sequence. Hence, the probability of drawing the sequence of a particular protein by random selection is $10^{-130}$. On the other hand, lattice simulations (Lau \& Dill, 1990) show that the probability of drawing any sequence which will fold to a specified structure (the ribonuclease configuration, for example), is estimated to be about 120 orders of magnitude larger than this.

hydrophobicity cannot be solely responsible for determining the single native structure. The hydrophobic contact free energies of the native and the next higher free energy level will, in general, differ by no more than one or two nonpolar contacts, less than $2 \mathrm{kcal} / \mathrm{mol}$. At this level of discrimination, the nondominant interactions are important. If they were not, then it would be impossible to account for the existence of hydrogen bonding, the partial successes of the intrinsic propensities, and the observed nonrandom distributions of charges, ion pairs, and aromatic groups (Burley \& Petsko, 1985, 1988; Blundell et al., 1986). Thus, hydrophobicity may "select" a relatively small number of compact conformations, from which the native structure is determined by the balance of all types of interactions.

\section{(10) Sequence Space and Origins of Proteins from RANDOM SEQUENCES}

That hydrophobicity is the dominant folding force is also consistent with expectations for the nature of mutation-induced changes in proteins. (1) Replacements of hydrophobic residues in the core are more disruptive of stability than other types of substitutions (Perutz \& Lehman, 1968; Reidhaar-Olson \& Sauer, 1988; Alber et al., 1987b; Alber et al., 1988; Lim \& Sauer, 1989; Lau \& Dill, 1990). (2) Because hydrophobicity is relatively nonspecific and orientation independent, many different core replacements are tolerated, provided only that they are hydrophobic and not significantly different in size. There is even greater tolerance for replacements of surface residues (Perutz \& Lehman, 1968; Lim \& Sauer, 1989; Bowie et al., 1990; Alber et al., 1987b; Lau \& Dill, 1990). (3) It follows that a large fraction of the molecules in "sequence space" (all the possible sequences) should be able to fold. Two-dimensional lattice simulations predict that more than $50 \%$ of all possible sequences will, under native conditions, fold to within $10 \%$ of the minimum possible radius of gyration and will therefore have considerable amounts of secondary structure (Lau \& Dill, 1989; Chan and Dill, unpublished results). White and Jacobs (1990) have found remarkably little difference between hydrophobicity sequence patterns in more than 8000 known sequences on the one hand and random sequences on the other. (4) There should be an extremely large number of "convergent" sequences: i.e., a given native structure should be encodable in many different sequences (Lau \& Dill, 1990; Chan and Dill, unpublished results). This implies a significant probability that a random sequence of amino acids will encode a globular conformation in general and a particular native structure in specific. For example, although there is only one sequence of ribonuclease, there should be more than $10^{100}$ different sequences which will all have the same native backbone conformation as ribonuclease (see Figure 14) (Lau \& Dill, 1990). Indeed, there has been considerable success in designing proteins based on simple principles (Richardson \& Richardson, 1987; DeGrado, 1988; DeGrado et al., 1989). The nonspecific nature of the dominant folding forces may therefore be essential in explaining how functional proteins could have originated from random sequences since only a negligible fraction of sequence space could have been sampled during the origins of life.

\section{CONCLUSIONS}

More than 30 years after Kauzmann's insightful hypothesis, there is now strong accumulated evidence that hydrophobicity is the dominant force of protein folding, provided that "hydrophobic" is operationally defined in terms of the transfer of nonpolar amino acids from water into a medium that is nonpolar and preferably capable of hydrogen bonding. Other forces are weaker but can affect stability. In acids and bases, electrostatic charge repulsions destabilize native proteins. Near neutral $\mathrm{pH}$, ion pairing can stabilize proteins. There is evidence that hydrogen bonding or van der Waals interactions among polar amino acids may be important, but their magnitude remains poorly understood. An important contributor to protein structure and stability is the dominant opposing force, arising principally from the loss of nonlocal conformational entropy due to steric contraints in the folded state. The marginal stabilities of proteins arise from the small difference between these large driving and opposing forces. Hydrophobicity leads to compact conformations with nonpolar cores, but it is the steric constraints in compact chains that are largely responsible for their considerable internal architecture. The reason that only one native structure is encoded in the amino acid sequence may be largely attributable to the hydrophobic interaction; there are only a small number of ways to configure a chain to maximize the number of nonpolar contacts. These forces are of a nature such that proteins should be tolerant of amino acid substitution, a given native structure should be encodable in many different sequences, and a large fraction of all possible sequences should fold to compact structured native states.

\section{ACKNOWLEDGMENTS}

I thank Tom Alber, Darwin Alonso, T. Arakawa, R. L. Baldwin, Rod Biltonen, Sarina Bromberg, Hue Sun Chan, Fred Cohen, Linda DeYoung, Gregg Fields, Rick Fine, Stan Gill, Barry Honig, Jack Kirsch, Peter Kollman, Tack Kuntz, Jim Lee, Kip Murphy, Nick Pace, George Rose, Jeff Skolnick, Dirk Stigter, Peter Wright, and Kai Yue for helpful discussions. I thank particularly Wayne Becktel for providing a very helpful list of early references and John Schellman for his unique and insightful perspective. Thanks to the NIH, the DARPA URI Program, and the Pew Scholars Foundation for support.

\section{REFERENCES}

Acampora, G., \& Hermans, J. (1967) J. Am. Chem. Soc. 89, 1543.

Ahmad, F., \& Bigelow, C. C. (1986) Biopolymers 25, 1623.

Alber, T. (1989a) Annu. Rev. Biochem. 58, 765.

Alber, T. (1989b) in Prediction of Protein Structure and the Principles of Protein Conformation (Fasman, G. D., Ed.) p 161, Plenum, New York.

Alber, T., et al. (1987a) Nature 330, 41.

Alber, T., et al. (1987b) Biochemistry 26, 3754.

Alber, T., et al. (1988) Science 239, 631.

Allen, G., Watkinson, J. G., \& Webb, K. H. (1966) Spectrochim. Acta 22, 807. 
Alonso, D. O. V., \& Dill, K. A. (1990) Biochemistry (submitted for publication).

Alvarez, J., \& Biltonen, R. (1973) Biopolymers 12, 1815.

Anderson, D. E., Becktel, W. J., \& Dahlquist, F. W. (1990) Biochemistry 29, 2403.

Anfinsen, C. B. (1973) Science 181, 223.

Anson, M. L. (1945) Adv. Protein Chem. 2, 361.

Anson, M. L., \& Mirsky, A. E. (1925) J. Gen. Physiol. 9, 169.

Anson, M. L., \& Mirsky, A. E. (1931) J. Phys. Chem. 35, 185.

Anson, M. L., \& Mirsky, A. E. (1934) J. Gen. Physiol. 17, 393.

Anufrieva, E. V., et al. (1968) J. Polym. Sci. 16, 3533.

Arakawa, T., \& Timasheff, S. N. (1982) Biochemistry 21, 6536.

Arakawa, T., \& Timasheff, S. N. (1984) Biochemistry 23, 5912.

Arakawa, T., Bhat, R., \& Timasheff, S. N. (1990) Biochemistry 29, 1924.

Argos, P. (1987) J. Mol. Biol. 197, 331.

Back, J. F., Oakenfull, D., \& Smith, M. B. (1979) Biochemistry 18, 5191.

Baker, E. N., \& Hubbard, R. E. (1984) Prog. Biophys. Mol. Biol. 44, 97.

Baldwin, R. L. (1986) Proc. Natl. Acad. Sci. U.S.A. 83, 8069.

Barlow, D. J., \& Thornton, J. M. (1983) J. Mol. Biol. 168, 867.

Bartlett, P. A., \& Marlowe, C. K. (1987) Science 235, 569.

Bashford, D., Chothia, C., \& Lesk, A. M. (1987) J. Mol. Biol. 196, 199.

Baum, J., et al. (1989) Biochemistry 28, 7.

Baumann, G., Frommel, C., \& Sander, C. (1989) Protein Eng. 2, 329.

Becktel, W., \& Schellman, J. (1987) Biopolymers 26, 1859.

Bendzko, P. I., Pfeil, W. A., Privalov, P. L., \& Tiktopulo, E. I. (1988) Biophys. Chem. 29, 301.

Blagdon, D. E., \& Goodman, M. (1975) Biopolymers 14, 241.

Blundell, T, et al. (1986) Science 234, 1005.

Bolen, D. W., \& Santoro, M. M. (1988) Biochemistry 27, 8069.

Bowie, J. U., Reidhaar-Olsen, J. F., Lim, W. A., \& Sauer, R. T. (1990) Science 247, 1306.

Bradley, E. K., Thomasen, J. F., Cohen, F. E., Kosen, P. A., \& Kuntz, I. D. (1990) J. Mol. Biol. (submitted for publication).

Brandts, J. F. (1964a) J. Am. Chem. Soc. 86, 4291.

Brandts, J. F. (1964b) J. Am. Chem. Soc. 86, 4302.

Brandts, J. F. (1969) in Structure and Stability of Biological Macromolecules (Timasheff, S. N., \& Fasman, G. D., Eds.) p 213, Dekker, New York.

Brandts, J. F., \& Lumry, R. (1963) J. Phys. Chem. 67, 1484.

Brandts, J. F., Oliveira, R. J., \& Westort, C. (1970) Biochemistry 9, 1038.

Brems, D. N., \& Havel, H. A. (1989) Proteins 5, 93.

Burley, S. K., \& Petsko, G. A. (1985) Science 229, 23.

Burley, S. K., \& Petsko, G. A. (1988) Adv. Protein Chem. $39,125$.

Burteau, N., Burton, S., \& Crichton, R. R. (1989) FEBS Lett. $258,185$.

Butler, J. A. V. (1937) Trans. Faraday Soc. 33, 229.

Chan, H. S., \& Dill, K. A. (1989a) J. Chem. Phys. 90, 492.

Chan, H. S., \& Dill, K. A. (1989b) Macromolecules 22, 4559.

Chan, H. S., \& Dill, K. A. (1990a) J. Chem. Phys. 92, 3118.

Chan, H. S., \& Dill, K. A. (1990b) Proc. Nat. Acad. Sci. U.S.A. (in press).
Chen, B., \& Schellman, J. A. (1989) Biochemistry 28, 685.

Chick, H., \& Martin, C. J. (1911) J. Physiol. 43, 1.

Chothia, C. (1974) Nature 254, 304.

Chothia, C. (1976) J. Mol. Biol. 105, 1.

Chou, P. Y., \& Fasman, G. D. (1978) Adv. Enzymol. 47, 45.

Chou, P. Y., Wells, M., \& Fasman, G. D. (1972) Biochemistry $11,3028$.

Christensen, J. K. (1952) C. R. Trav. Lab. Carlsberg, Ser. Chim. 28, 37.

Christian, S. D., \& Tucker, E. E. (1982) J. Solution Chem. $11,749$.

Cohn, E. J., et al. (1933) J. Biol. Chem. 100, 3.

Collins, K. D., \& Washabaugh, M. W. (1985) Q. Rev. Biophys. 18, 323.

Conio, G., Patrone, E., \& Brighetti, S. (1970) J. Biol. Chem. 245, 3335 .

Covell, D. G., \& Jernigan, R. L. (1990) Biochemistry 29, 3287-3294.

Crovetto, R., Fernandez-Priri, R., \& Japas, M. L. (1982) J. Chem. Phys. 76, 1077.

Cybulski, S., M., \& Scheiner, S. (1989) J. Phys. Chem. 93, 6565.

Dang, L. X., Merz, K. M., \& Kollman, P. A. (1989) J. Am. Chem. Soc. 111, 8505 .

Debye, P. (1949) Ann. N.Y. Acad. Sci. 51, 575.

deGennes, P. G. (1975) J. Phys. (Paris) 36, L-55.

deGennes, P. G. (1979) in Scaling Concepts in Polymers Physics, Cornell University Press, Ithaca, NY.

DeGrado, W. F. (1988) Adv. Protein Chem. 39, 51.

DeGrado, W. F., Wasserman, Z. R., \& Lear, J. D. (1989) Science 243, 622.

Dill, K. A. (1985) Biochemistry 24, 1501.

Dill, K. A. (1990) Science (in press).

Dill, K. A., \& Alonso, D. O. V. (1988) in Colloquium Mosbach der Gessellschaft fur Biologische Chemie: Protein Structure and Protein Engineering (Huber, T., \& Winnacker, E. L., Eds.) Vol. 39, p 51, Springer-Verlag, Berlin.

Dill, K. A., Alonso, D. O. V., \& Hutchinson, K. (1989) Biochemistry 28, 5439.

Doty, P., \& Yang, J. T. (1956) J. Am. Chem. Soc. 78, 498.

Doty, P., et al. (1954) J. Am. Chem. Soc. 76, 4493.

Doty, P., Bradbury, J. H., \& Holtzer, A. M. (1956) J. Am. Chem. Soc. 78, 947.

Doty, P., et al. (1958) Proc. Natl. Acad. Sci. U.S.A. 44, 424.

Dyson, H. J., et al. (1985) Nature 318, 480.

Dyson, H. J., et al. (1988a) J. Mol. Biol. 201, 161.

Dyson, H. J., et al. (1988b) J. Mol. Biol. 201, 201.

Edelhoch, H., \& Osborne, J. C. (1976) Adv. Protein Chem. 30, 183.

Eden, D., et al. (1982) Proc. Natl. Acad.Sci. U.S.A. 79, 815.

Edsall, J. T. (1935) J. Am. Chem. Soc. 57, 1506.

Edsall, J. T., \& McKenzie, H. A. (1983) Adv. Biophys. 16, 53.

Eisenberg, D., \& McLachlan, A. D. (1986) Nature 319, 199.

Eisenberg, M. A., \& Schwert, G. W. (1951) J. Gen. Physiol. 34,583 .

Ellis, R. J. (1988) Nature 328, 378.

Epand, R. M., \& Scheraga, H. A. (1968) Biochemistry 7, 2864.

Evans, P. A., et al. (1987) Nature 329, 266.

Eyring, H., \& Stearn, A. E. (1939) Chem. Rev. 24, 253.

Fahey, P. F., Krupke, D. W., \& Beams, J. W. (1969) Proc. Natl. Acad. Sci. U.S.A. 63, 548.

Fauchere, J.-L., \& Pliska, V. E. (1983) Eur. J. Med. Chem.-Chem. Therm. 18, 369. 
Fersht, A. R. (1972) J. Mol. Biol. 64, 497.

Fersht, A. R. (1985) Nature 314, 235.

Finkelstein, A. V., \& Ptitsyn, O. B. (1987) Prog. Biophys. Mol. Biol. 50, 171.

Flory, P. J. (1953) in Principles of Polymer Chemistry, Cornell University Press, Ithaca, NY.

Flory, P. J. (1969) Statistical Mechanics of Chain Molecules, Wiley, New York.

Frank, H. S., \& Evans, M. W. (1945) J. Chem. Phys. 13, 507.

Franks, F. (1983) Water, The Royal Society of Chemistry, London.

Franzen, J. S., \& Stephens, R. E. (1963) Biochemistry 2, 1321.

Freed, K. F. (1987) in Renormalization Group Theory of Macromolecules, Wiley, New York.

Friedman, M. E., \& Scheraga, H. A. (1965) J. Phys. Chem. 69, 3795.

Friend, S. H., \& Gurd, F. R. N. (1979) Biochemistry 18, 4612.

Gavish, B., Gratton, E., \& Hardy, C. J. (1983) Proc. Natl. Acad. Sci. U.S.A. 80, 750.

Geiger, A., Rahman, A., \& Stillinger, F. H. (1979) J. Chem. Phys. 70, 263-276.

Gekko, K., \& Timasheff, S. N. (1981) Biochemistry 20, 4667.

Gibbs, J. H., \& DiMarzio, E. A. (1959) J. Chem. Phys. 30 , 271.

Gill, S. J., \& Noll, L. (1972) J. Phys. Chem. 76, 3065.

Gill, S. J., \& Wadsö, I. (1976) Proc. Natl. Acad. Sci. U.S.A. 73, 2955.

Gill, S. J., et al. (1985) J. Phys. Chem. 89, 3758.

Gilson, M. K., \& Honig, B. H. (1988a) Proteins 3, 32.

Gilson, M. K., \& Honig, B. H. (1988b) Proteins 4, 7.

Gilson, M. K., \& Honig, B. H. (1989) Proc. Natl. Acad. Sci. U.S.A. 86, 1524.

Goldenberg, D. P., \& Creighton, T. E. (1983) J. Mol. Biol. $165,407$.

Goodman, M., et al. (1969) Proc. Natl. Acad. Sci. U.S.A. 64, 444.

Goto, Y., \& Fink, A. L. (1989) Biochemistry 28, 945.

Goto, Y., \& Fink, A. L. (1990) J. Mol. Biol. (in press).

Goto, Y., Calciano, L. J., \& Fink, A. L. (1990) Proc. Natl. Acad. Sci. U.S.A. 87, 573.

Griko, Y. V., Privalov, P. L., Sturtevant, J. M., \& Venyamenov, S. Y. (1988) Proc. Natl. Acad. Sci, U.S.A. 85, 3343.

Grutter, M. G., et al. (1987) J. Mol. Biol. 197, 315.

Gurney, R. W. (1962) in Ionic Processes in Solution, Dover, New York.

Guy, H. R. (1985) Biophys. J. 47, 61.

Ha, J. H., Spolar, R. S., \& Record, M. T., Jr. (1989) J. Mol. Biol. 209, 801.

Harrington, W. F., \& Schellman, J. A. (1956) C. R. Lab. Carlsberg, Ser. Chim. 30, 21.

Haschemeyer, R. H., \& Haschemeyer, A. E. V. (1973) in Proteins, Wiley, New York.

Hawley, S. A. (1971) Biochemistry 10, 2436.

Hermans, J. (1966) J. Am. Chem. Soc. 88, 2418.

Hermans, J., \& Scheraga, H. A. (1961) J. Am. Chem. Soc. $83,3283$.

Hildebrand, J. H. (1968) J. Phys. Chem. 72, 1841.

Hildebrand, J. H. (1979) Proc. Natl. Acad. Sci. U.S.A. 76, 194.

Hildebrand, J. H., \& Scott, R. L. (1950) in The Solubility of Nonelectrolytes, Reinhold, New York.

Hill, T. L. (1960) in Introduction to Statistical Thermodynamics, Addison-Wesley, Reading, MA.

Hollecker, M., \& Creighton, T. E. (1982) Biochim. Biophys. Acta $701,395$.
Holley, L. H., \& Karplus, M. (1989) Proc. Natl. Acad. Sci. U.S.A. 86, 152.

Honig, B., \& Hubbell, W. (1984) Proc. Natl. Acad. Sci. U.S.A. 81,5412 .

Honig, B., Hubbell, W., \& Flewelling, R. F. (1986) Annu. Rev. Biophys. Biophys. Chem. 15, 163.

Hvidt, A. (1975) J. Theor. Biol. 50, 245.

Jacobsen, C. F., \& Linderstrom-Lang, K. (1949) Nature 164, 411.

Janin, J., et al. (1978) J. Mol. Biol. 125, 357.

Jolicoeur, C., et al. (1986) J. Solution Chem. 15, 109.

Jorgensen, W. L. (1989) Acc. Chem. Res. 22, 184.

Jorgensen, W. L., Geo, J., \& Ravimohan, C. (1985) J. Phys. Chem. 89, 3470.

Kabsch, W., \& Sander, C. (1983) Biopolymers 22, 2577.

Kabsch, W., \& Sander, C. (1984) Proc. Natl. Acad. Sci. U.S.A. 1075.

Karle, I. L., et al. (1990) Proteins 7, 62.

Karplus, M., Ichiye, T., \& Pettitt, B. M. (1987) Biophys. J. 52, 1083.

Kauzmann, W. (1954) in The Mechanism of Enzyme Action (McElroy, W. D., \& Glass, B., Eds.) p 70, Johns Hopkins Press, Baltimore, MD.

Kauzmann, W. (1959) Adv. Protein Chem. 14, 1.

Kauzmann, W. (1987) Nature 325, 763.

Kellis, J. T., Nyberg, K., \& Fersht, A. R. (1989) Biochemistry $28,4914$.

Kelly, L., \& Holladay, L. A. (1987) Protein Eng. I, 137.

Kendrew, J. C. (1961) Sci. Am. 205, (6), 96.

Kendrew, J. C., et al. (1958) Nature 181, 662.

Kim, P. S., \& Baldwin, R. L. (1984) Nature 307, 329.

Klapper, M. H. (1971) Biochim. Biophys. Acta 229, 557.

Klotz, I. M., \& Franzen, J. S. (1962) J. Am. Chem. Soc. 84, 3461 .

Klotz, I. M., \& Farnham, S. B. (1968) Biochemistry 7, 3879.

Kneller, D. G., Cohen, F. E., \& Langridge, R. (1990) J. Mol. Biol. (in press).

Kresheck, G. C., \& Klotz, I. M. (1969) Biochemistry 8, 8.

Krikorian, S. E. (1982) J. Phys. Chem. 86, 1875.

Kundrot, C. E., \& Richards, F. M. (1987) J. Mol. Biol. 193 , 157.

Kuntz, I. D. (1972) J. Am. Chem. Soc. 94, 4009.

Kuwajima, K. (1989) Proteins 6, 87.

Kyte, J., \& Doolittle, R. F. (1982) J. Mol. Biol. 157, 105.

Lau, K. F., \& Dill, K. A. (1989) Macromolecules 22, 3986.

Lau, K. F., \& Dill, K. A. (1990) Proc. Natl. Acad. Sci. U.S.A. 87,638 .

Lee, B. K., \& Richards, F. M. (1971) J. Mol. Biol. 55, 379.

Lee, J. C., \& Timasheff, S. N. (1981) J. Biol. Chem. 256, 7193.

Lennard-Jones, J., \& Pople, J. A. (1951) Proc. R. Soc. London A205, 155.

Levitt, M., \& Greer, J. (1977) J. Mol. Biol. 114, 181.

Lifschitz, I. M. (1968) Zh. Eksp. Teor. Fiz. 55, 2408.

Lim, W. A., \& Sauer, R. T. (1989) Nature 339, 31.

Linderstrom-Lang, K. U. (1924) C. R. Trav. Lab. Carlsberg, 15,70 .

Linderstrom-Lang, K. U. (1952) Lane Medical Lectures, Vol. 6, p 53, Stanford University Press, Stanford, CA.

Lotan, N., Bixon, M., \& Berger, A. (1967) Biopolymers 5, 69.

Luger, K., et al. (1989) Science 243, 206.

Lumry, R., \& Eyring, H. (1954) J. Phys. Chem. 58, 110.

Lumry, R., Biltonen, R., \& Brandts, J. F. (1966) Biopolymers $4,917$. 
Lupu-Lotan, N., Yaron, A., Berger, A., \& Sela, M. (1965) Biopolymers 3, 625.

Marqusee, S., \& Baldwin, R. L. (1987) Proc. Natl. Acad. Sci. U.S.A. 84, 8898 .

Marqusee, S., Robbins, V. H., \& Baldwin, R. L. (1989) Proc. Natl. Acad. Sci. U.S.A. 86, 5286.

Masterson, W. L. (1954) J. Chem. Phys. 22, 1830.

Matsumura, M., Becktel, W. J., \& Matthews, B. W. (1988a) Nature 334, 406.

Matsumura, M., et al. (1988b) Eur. J. Biochem. 171, 715.

Matsumura, M., Matthews, B. W., Levitt, M., \& Becktel, W. J. (1989) Proc. Natl. Acad. Sci. U.S.A. 86, 6562.

Matthew, J. B., \& Richards, F. M. (1982) Biochemistry 21, 4489.

Matthew, J. B., \& Gurd, F. R. N. (1986) Methods Enzymol. $130,413$.

Matthews, B. W. (1987a) Harvey Lect. 81, 33.

Matthews, B. W. (1987b) Biochemistry 26, 6885 .

Matthews, B. W., Nicholson, A., \& Becktel, W. J. (1987) Proc. Natl. Acad. Sci. U.S.A. 84, 6663.

Mattice, W. L., \& Scheraga, H. A. (1984) Biopolymers 23, 1701.

McGregor, M. J., Islam, S. A., \& Sternberg, M. J. E. (1987) J. Mol. Biol. 198, 295.

Meirovitch, H., \& Scheraga, H. A. (1980) Macromolecules 13, 1406.

Merutka, G., \& Stellwagen, E. (1990) Biochemistry 29, 894.

Merutka, G., et al. (1990) Biochemistry (submitted for publication).

Miller, K. W., \& Hildebrand, J. H. (1968) J. Am. Chem. Soc. 90, 3001.

Mirsky, A. E., \& Pauling, L. (1936) Proc. Natl. Acad. Sci. U.S.A. 22, 439.

Mitchinson, C., \& Baldwin, R. L. (1986) Proteins 1, 23.

Miyazawa, S., \& Jernigan, R. L. (1985) Macromolecules 18 , 534.

Morrison, T. J. (1952) J. Chem. Soc. 3, 3814.

Morrison, T. J., \& Billett, F. (1952) J. Chem. Soc. 3, 3819.

Muller, N. (1990) Acc. Chem. Res. 23, 23.

Murphy, K. P., \& Gill, S. J. (1989a) J. Chem. Thermodyn. $21,903$.

Murphy, K. P., \& Gill, S. J. (1989b) Thermochim. Acta 139, 279.

Murphy, K. P., Privalov, P. L., \& Gill, S. J. (1990) Science $247,559$.

Nelson, J. W., \& Kallenbach, N. R. (1986) Proteins 1, 211.

Nemethy, G., \& Scheraga, H. A. (1962) J. Chem. Phys. 36, 3401 .

Nemethy, G., Scheraga, H. A., \& Kauzmann, W. (1968) J. Phys. Chem. 72, 1842.

Nemethy, G., Peer, W. J., \& Scheraga, H. A. (1981) Annu. Rev. Biophys. Bioeng. 10, 459.

Neurath, H., et al. (1944) Chem. Rev. 34, 157.

Nojima, H., et al. (1977) J. Mol. Biol. 116, 429.

Northrup, J. H. (1932) J. Gen. Physiol. 16, 323.

Novotny, J., Bruccoleri, R. E., \& Karplus, M. (1984) J. Mol. Biol. 177, 787.

Novotny, J., Rashin, A. A., \& Bruccoleri, R. E. (1988) Proteins 4, 19.

Nozaki, Y., \& Tanford, C. (1970) J. Biol. Chem. 245, 1698.

Nozaki, Y., \& Tanford, C. (1971) J. Biol. Chem. 246, 2211.

Ooi, T., \& Oobatake, M. (1988) J. Biochem. (Tokyo) 103, 114.

Ostermann, J., et al. (1989) Nature 341, 125.
Pace, C. N. (1975) CRC Crit. Rev. Biochem. 3, 1.

Pace, C. N., \& Tanford, C. (1968) Biochemistry 7, 198.

Pace, C. N., \& Grimsley, G. R. (1988) Biochemistry 27, 3242.

Pace, C. N., Grimsley, G. R., Thomson, J. A., \& Barnett, B. J. (1988) J. Biol. Chem. 263, 11820.

Padmanabhan, S., et al. (1990) Nature 344, 268.

Pangali, C., Rao, M., \& Berne, B. J. (1982) J. Chem. Phys. 71, 2982.

Parodi, R. M., Bianchi, E., \& Ciferri, A. (1973) J. Biol. Chem. $248,4047$.

Pauling, L. (1960) in The Nature of the Chemical Bond, 3rd ed., Cornell University Press, Ithica, NY.

Pauling L., \& Corey, R. B. (1951a) Proc. Natl. Acad. Sci. U.S.A. 37, 235.

Pauling, L., \& Corey, R. B. (1951b) Proc. Natl. Acad. Sci. U.S.A. 37, 251.

Pauling, L., \& Corey, R. B. (1951c) Proc. Natl. Acad. Sci. U.S.A. 37, 272.

Pauling, L., \& Corey, R. B. (1951d) Proc. Natl. Acad. Sci. U.S.A. 37, 729.

Pauling, L., Corey, R. B., \& Branson, H. R. (1951) Proc. Natl. Acad. Sci. U.S.A. 37, 205.

Peller, L. (1959) J. Phys. Chem. 63, 1194.

Perutz, M. F., \& Lehmann, H. (1968) Nature 219, 902.

Perutz, M. F., \& Raidt, H. (1975) Nature 255, 256.

Perutz, M. F., Kendrew, J. C., \& Watson, H. C. (1965) J. Mol. Biol. 13, 669 .

Piela, L., Nemethy, G., \& Scheraga, H. A. (1987) Biopolymers 26, 1273.

Platzer, K. E. B., et al. (1972) Macromolecules 5, 177.

Poland, D. C., \& Scheraga, H. A. (1965) Biopolymers 3, 379.

Poland, D. C., \& Scheraga, H. A. (1970) Theory of the Helix-Coil Transition, Academic Press, New York.

Ponder, J. W., \& Richards, F. M. (1987) J. Mol. Biol. 193, 775.

Post, C. B., \& Zimm, B. H. (1979) Biopolymers 18, 1487.

Presnell, S. R., \& Cohen, F. E. (1989) Proc. Natl. Acad. Sci. U.S.A. 86, 6592.

Privalov, P. L. (1979) Adv. Protein Chem. 33, 167.

Privalov, P. L. (1989) Annu. Rev. Biophys. Biophys. Chem. 18, 47.

Privalov, P. L., \& Kechinashvili, N. N. (1974) J. Mol. Biol. 86, 665.

Privalov, P. L., \& Gill, S. J. (1988) Adv. Protein Chem. 39, 191.

Privalov, P. L., et al. (1986) J. Mol. Biol. 190, 487.

Privalov, P. L., Gill, S. J., \& Murphy, K. P. (1990) Science (in press).

Ptitsyn, O. B. (1987) J. Protein Chem. 6, 273.

Qian, N., \& Sejnowski, T. J. (1988) J. Mol. Biol. 202, 865.

Radzicka, A., \& Wolfenden, R. (1988) Biochemistry 27, 1664.

Rao, S. P., Carlstrom, D. E., \& Miller, W. G. (1974) Biochemistry 13, 943.

Ravishanker, G., Mezei, M., \& Beveridge, D. L. (1982) Faraday Symp. Chem. Soc. 17, 79.

Rich, D. H., \& Jasensky, R. D. (1980) J. Am. Chem. Soc. 102, 1112 .

Richards, F. M. (1977) Annu. Rev. Biophys. Bioeng. 6, 151.

Richards, F. M., \& Richmond, T. (1978) in Molecular Interactions and Activity in Proteins, (Wolstenholme, G. E., Ed.) p 23, Ciba Foundation Symposium 60, Excerpta Medica, Amsterdam. 
Richardson, J. S., \& Richardson, D. C. (1987) in Protein Engineering (Oxender, D. L., \& Fox, C. F., Eds.) p 149, Alan R. Liss, New York.

Richardson, J. S., \& Richardson, D. C. (1988) Science 240 , 1648.

Robertson, T. B. (1918) The Physical Chemistry of the Proteins, Longmans, Green and Co., New York.

Rogers, N. K. (1989) in Prediction of Protein Structure and the Principles of Protein Conformation (Fasman, G. D., Ed.) p 359, Plenum, New York.

Rooman, M. J., \& Wodak, S. J. (1988) Nature 335, 45.

Rose, G. D., et al. (1985a) Science 229, 834.

Rose, G. D., Gierasch, L. M., \& Smith, J. A. (1985b) Adv. Protein Chem. 37, 1.

Roseman, M. A. (1988) J. Mol. Biol. 201, 621.

Rothman, J. E. (1989) Cell 59, 591.

Sanchez, I. C. (1979) Macromolecules 12, 980.

Sandberg, W. S., \& Terwilliger, T. C. (1989) Science 245 , 54.

Santoro, M. M., \& Bolen, D. W. (1988) Biochemistry 27, 8063.

Scheiner, S., \& Hillenbrand, E. A. (1985) Proc. Natl. Acad. Sci U.S.A. 82, 2741.

Scheiner, S., Redfern, P., \& Hillenbrand, E. A. (1986) Int. J. Quant. Chem. 29, 817.

Schellman, C., \& Schellman, J. A. (1958) C. R. Lab. Carlsberg, Ser. Chim. 30, 463.

Schellman, J. A. (1955) C. R. Trav. Lab. Carlsberg, Ser. Chim. 29, 223.

Schellman, J. A. (1958a) J. Phys. Chem. 62, 1485.

Schellman, J. A. (1958b) C.R. Trav. Lab. Carlsberg, Ser. Chim. 30, 450 .

Schrier, M. Y., \& Schrier, E. E. (1976) Biochemistry 15, 2607.

Schulz, G. E., \& Schirmer, R. H. (1979) in Principles of Protein Structure, Springer-Verlag, New York.

Semlyen, J. A. (1976) in Advances in Polymer Science (Cantow, H. J., et al., Eds.) Vol. 21, Springer, Berlin.

Shakhnovich, E. I., \& Finkelstein, A. V. (1989a) Biopolymers $28,1667$.

Shakhnovich, E. I., \& Finkelstein, A. V. (1989b) Biopolymers 28,1681 .

Shoemaker, K. R., et al. (1985) Proc. Natl. Acad. Sci. U.S.A. $82,2349$.

Shoemaker, K. R., et al. (1987) Nature 326, 563.

Shoemaker, K. R., et al. (1990) Biopolymers 29, 1.

Shortle, D., \& Meeker, A. K. (1989) Biochemistry 28, 936.

Shortle, D., Meeker, A. K., \& Freire, E. (1988) Biochemistry 27,4761 .

Sikorski, A., \& Skolnick, J. (1989) Proc. Natl. Acad. Sci. U.S.A. 86, 2668.

Singer, S. J. (1962) Adv. Protein Chem. 17, 1.

Skolnick, J., Kolinski, A., \& Yaris, R. (1988) Proc. Natl. Acad. Sci. U.S.A. 85, 5057.

Skolnick, J., Kolinski, A., \& Yaris, R. (1989) Proc. Natl. Acad. Sci. U.S.A. 86, 1229.

Sneddon, S. F., Tobias, D. J., \& Brooks, C. L., III (1989) J. Mol. Biol. 209, 817.
Srinivasan, R. (1976) Indian J. Biochem. Biophys. 13, 192.

Stigter, D., \& Dill, K. A. (1989) J. Phys. Chem. 93, 6737.

Stigter, D., \& Dill, K. A. (1990) Biochemistry 29, 1262.

Stigter, D., Alonso, D. O. V., \& Dill, K. A. (1990) (submitted for publication).

Stillinger, F. H. (1980) Science 209, 451.

Sturtevant, J. M. (1977) Proc. Natl. Acad. Sci. U.S.A. 74, 2236.

Sueki, M., et al. (1984) Macromolecules 17, 148.

Susi, H. (1969) in Structure and Stability of Biological Macromolecules (Timasheff, S. N., \& Fasman, G. D., Eds.) Marcel Dekker, New York.

Susi, H., \& Ard, J. S. (1969) J. Phys. Chem. 73, 2440.

Sweet, R. M., \& Eisenberg, D. (1983) J. Mol. Biol. 171, 479.

Tadokoro, H. (1979) in Structure of Crystalline Polymers, Wiley, New York.

Tanford, C. (1961) in Physical Chemistry of Macromolecules, Chapter 7, Wiley, New York.

Tanford, C. (1962) J. Phys. Chem. 84, 4240.

Tanford, C. (1968) Adv. Protein Chem. 23, 121.

Tanford, C. (1970) Adv. Protein Chem. 24, 1.

Tanford, C. (1979) Proc. Natl. Acad. Sci. U.S.A. 76, 4175.

Tanford, C. (1980) in The Hydrophobic Effect, 2nd ed., Wiley, New York.

Tanford, C., \& Kirkwood, J. G. (1957a) J. Am. Chem. Soc. $79,5333$.

Tanford, C., \& Kirkwood, J. G. (1957b) J. Am. Chem. Soc. $79,5340$.

Taniuchi, H., \& Anfinsen, C. B. (1969) J. Biol. Chem. 244, 3864.

Thornton, J. (1988) Nature 335, 10.

Vinogradov, S. N., \& Linnell, R. H. (1971) in Hydrogen Bonding, Van Nostrand Reinhold, New York.

von Hippel, P. H., \& Schleich, T. (1969a) in Structure and Stability of Biological Macromolecules (Timasheff, S., \& Fasman, G., Eds.) Vol. II, p 417, Dekker, New York.

von Hippel, P. H., \& Schleich, T. (1969b) Acc. Chem. Res. $2,257$.

Wada, A., \& Nakamura, H. (1981) Nature 293, 757.

Weiner, S., et al. (1984) J. Am. Chem. Soc. 106, 765.

Wertz, D. H., \& Scheraga, H. A. (1978) Macromolecules 11, 9.

White, S. H., \& Jacobs, R. E. (1990) Biophys. J. (in press).

Wright, P. E., Dyson, H. J., \& Lerner, R. A. (1988) Biochemistry 27, 7167.

Wu, H. (1929) Am. J. Physiol. 90, 562.

Wu, H. (1931) Chin. J. Physiol. 5, 321.

Wu, H., \& Wu, D. (1931) Chin. J. Physiol. 5, 369.

Yutani, K., et al. (1984) J. Biol. Chem. 259, 14076.

Yutani, K., et al. (1987) Proc. Natl. Acad. Sci. U.S.A. 84, 4441.

Zhang, R., \& Snyder, G. H. (1989) J. Biol. Chem. 264, 18,472 .

Zimm, B. H., \& Bragg, J. K. (1959) J. Chem. Phys. 31, 526.

Zimm, B. H., \& Rice, S. A. (1960) Mol. Phys. 3, 391.

Zimm, B. H., et al. (1959) Proc. Natl. Acad. Sci. U.S.A. 45, 1601.

Zipp, A., \& Kauzmann, W. (1973) Biochemistry 12, 4217. 